\title{
Análisis comparado de las especies del género Bryconamericus (Teleostei: Characidae) en la cuenca de los ríos Cauca-Magdalena y Ranchería, Colombia
}

\author{
Comparative analysis of species of the genus Bryconamericus (Teleostei: Characidae) from \\ Cauca, Magdalena and Ranchería basin rivers in Colombia
}

\author{
César Román-Valencia ${ }^{1 *}$, James A. Vanegas-Ríos ${ }^{1}$ y María D. García G. ${ }^{2}$ \\ ${ }^{1}$ Universidad del Quindio, Laboratorio de Ictiología, A. A. 2639, Armenia, Colombia. \\ ${ }^{2}$ Universidad del Quindio, Grupo de Investigación y Asesoría en Estadística, Armenia, Colombia. \\ *Correspondencia: ceroman@uniquindio.edu.co
}

Resumen. Se analizan las especies de Bryconamericus Eigenmann 1907 para la cuenca de los ríos Magdalena-Cauca y Ranchería, con base en material tipo y no tipo; además se establece la distribución geográfica de B. caucanus. Se emplean caracteres merísticos, morfométricos y osteológicos para definir la variación morfológica entre las especies de Bryconamericus analizadas. La forma de los Bryconamericus se estudió mediante un análisis de componentes principales (ACP), donde las medidas fueron previamente transformadas para corregir el efecto de talla y alometría. El número de vértebras diferenció a B. caucanus de otras especies del río Magdalena (37-39 vs. 40-42). Se encontró que B. arilepis Román-Valencia, Vanegas-Ríos and Ruiz-C. 2008 se distingue de los demás Bryconamericus del río Magdalena por las escamas en la línea lateral (50-54 vs. 34-50) y las escamas entre el origen de la aleta dorsal y la línea lateral (9-11 vs. 58). El índice de forma (IF) construido a partir del ACP confirmó las especies que comparten el mismo perfil: $B$. caucanus Eigenmann 1913 y B. plutarcoi Román-Valencia 2001, B. tolimae Eigenmann 1913, B. huilae Román-Valencia 2003, B. arilepis y Bryconamericus sp. nov. (en prensa), ambos grupos distinguibles entre sí. El análisis de varianza demostró que existen diferencias significativas entre las longitudes de las aletas pélvicas entre las especies de Bryconamericus $(\mathrm{F}=44,7$; $\mathrm{p}=0,000)$. Se observaron significancias en las longitudes de la aleta anal, al confrontar las especies de Bryconamericus analizadas $(\mathrm{F}=44,03 ; \mathrm{p}=0,000)$. Las poblaciones de $B$. caucanus de la parte baja del río Cauca son las que poseen mayor afinidad en forma con las demás poblaciones del río Ranchería, alto y medio Cauca y bajo Magdalena. Se determina que Hemibrycon dentatus y $H$. decurrens son sinónimos modernos de B. caucanus; se confirma la existencia de 2 especies nuevas para el río Sogamoso, cuenca del río Magdalena, y se establece la distribución geográfica de $B$. caucanus en la cuenca de los ríos Sinú, Ranchería, Cauca y bajo Magdalena.

Palabras clave: taxonomía, pez tropical, poblaciones, variación geográfica, Bryconamericus.

\begin{abstract}
We reviewed Bryconamericus species from the Cauca, Magdalena and Ranchería rivers in Colombia using type and new specimens. The geographic distribution of B.caucanus was determined. We determined the morphological variation among Bryconamericus species analyzed using meristic, morphometric and osteological characters. The shape of Bryconamericus was analyzed using principal component analysis (PCA), in which measurements were previously processed to correct for the effect of size. The number of vertebrae differentiated B. caucanus from other species of the Magdalena River (37-39 vs.40-42). Bryconamericus arilepis Roman-Valencia et al 2008 differs from other Río Magdalena Bryconamericus in lateral-line scales (50-54 vs. 34-50) and scales between dorsal-fin origin and lateral line (9-11 vs. 5-8). The index of form (IF) built with PCA confirmed the validity of species that share the same shape: $B$. caucanus Eigenmann 1913 and B. plutarcoi Roman-Valencia 2001, B. tolimae Eigenmann 1913, B. huilae Roman-Valencia 2003, B. arilepis and Bryconamericus sp. nov. Román-Valencia, Vanegas-Ríos and Ruiz-C. in press, and the 2 groups were distinguishable from each other. An ANOVA showed significant differences in pelvic-fin length among species of Bryconamericus $(\mathrm{F}=$ 44.7, $\mathrm{p}=0000)$. Anal-fin length was also useful to distinguish the studied species $(\mathrm{F}=44.03, \mathrm{p}=0000)$. Populations of B. caucanus from the lower Río Cauca show greatest similarity in shape with all other populations from Río Ranchería, upper and lower Cauca, and middle Magdalena. Hemibrycon dentatus and H. decurrens are shown to be synonyms of $B$. caucanus. We reconfirm the existence of 2 new species of Bryconamericus from Río Sogamoso, in the Río Magdalena basin. The geographical distribution of B. caucanus in the Sinú, Ranchería, Cauca and lower Magdalena river basins is established.
\end{abstract}

Key words: taxonomy, tropical fish, populations, geographic variation, Bryconamericus. 


\section{Introducción}

Dentro de los Characidae, el género Bryconamericus Eigenmann 1907 registra alta riqueza, con 78 especies (número que puede aumentar), incluye a Knodus Eigenmann 1911 (Román-Valencia, 2003a, RománValencia et al., 2008a), es de tamaño pequeño y se distribuye desde América Central (en Costa Rica y Panamá) hasta el oeste de Argentina, en una variedad de ecosistemas dulceacuícolas (Vari y Siebert, 1990; Miquelarena y Aquino, 1995; Langeani et al., 2005; Román-Valencia, 2002, 2003a, 2003b, 2004, 2005; Lima et al., 2003, 2004; Silva, 2004; Román-Valencia et al., 2008b, Vanegas-Ríos, 2008).

Para Colombia se reconocen 22 especies de Bryconamericus (Román-Valencia, 2003a; 2004; RománValencia et al., 2008b): B. alpha Eigenmann Henn et Wilson 1914, B. andresoi Román-Valencia 2003, $B$. carlosi Román-Valencia 2003, B. caucanus Eigenmann 1913, B. cismontanus Eigenmann, Henn and Wilson1914, B. cristiani Román-Valencia 1998, B. dahli RománValencia 2000, B. emperador Eigenmann and Ogle 1907, B. galvisi Román-Valencia2000, B. guaytarae Eigenmann, Henn and Wilson 1914, B. guizae Román-Valencia 2003, $B$. heteresthes Eigenmann1908, B. huilae Román-Valencia 2003, B. hypopterus Fowler 1943, B. ichoensis RománValencia 2000, B. loisae Géry 1964, B. miraensis Fowler 1945, B. multiradiatus Dahl 1960, B. orteguasae Fowler 1943, B. plutarcoi Román-Valencia 2001, B. peruanus Müller and Troschel 1845 y $B$. tolimae Eigenmann 1913. Además de 2 nuevas especies para la cuenca del río Magdalena (Román-Valencia et al., 2008b; RománValencia et al., en prensa).

Una de las especies de Bryconamericus en Colombia que registra amplia distribución es $B$. caucanus (RománValencia, 2003a), ha sido incluida en revisiones, filogenia (Eigenmann, 1927; Román-Valencia, 2003a; VanegasRíos, 2008 ), de composición y estructura (RománValencia, 1993; Cardona et al., 1998; Jiménez et al., 1998; Román-Valencia et al., 1999), ecología trófica y reproductiva (Román-Valencia y Muñoz, 2001; RománValencia et al., 2008c). Sin embargo, aún es confusa su distribución; por ejemplo, se registra para el alto Meta (Géry, 1977), Amazonia (Eigenmann y Allen, 1942; Fowler, 1948; Miles, 1947; Ortega y Vari, 1986; Barriga, 1991) y se ha sugerido su sinonimia con $B$. peruanus en el Pacífico ecuatoriano (Tortonose, 1941, 1942; Böhlke, 1958). Además, para igual localidad típica de esta especie en el alto Cauca, se describe a H. dentatus Eigenmann 1913 (Eigenmann, 1913), forma que nunca se ha colectado en el alto Cauca (Miles, 1971, 1973; Román-Valencia, 1993, 1995) y se registra para el alto Meta (Eigenmann, 1922,
1927) y aún para la isla de Trinidad (Price, 1955). Igual ocurre para $H$. decurrens Eigenmann 1913 (Eigenmann, 1913). Por lo tanto, el propósito de este artículo es determinar y comparar las especies de Bryconamericus para la cuenca de los ríos Cauca-Magdalena y Ranchería, con base en material fresco tipo y no tipo. De esta manera, se pretende resolver un problema taxonómico existente para los Bryconamericus de los drenajes mencionados

\section{Materiales y métodos}

Se analizaron datos de B. caucanus, B. tolimae, $B$. plutarcoi, $B$. huilae y de Bryconamericus recientemente descritas, identificadas como B. arilepis y Bryconamericus sp. nov. (Román-Valencia et al., 2008a; Román-Valencia et al., en prensa). Se hizo una comparación poblacional para establecer la distribución geográfica de B. caucanus con datos de su localidad típica y de diversas localidades a lo largo de la cuenca del río Magdalena y del río Ranchería, drenajes en la actualidad independientes (adicionalmente se usó información obtenida por Román-Valencia, 2003a). Para comprobar la validez de $H$. dentatus y $H$. decurrens se incluyó material tipo. Las acronimias de los museos siguieron a Leviton et al. (1985), con la adición del Laboratorio de Ictiología, Universidad del Quindío, Armenia, Colombia (IUQ) (véase material examinado, Apéndice 1).

Variables. Siguiendo a Fink y Weitzman (1974), se hicieron conteos y se tomaron medidas (Cuadro 1) sobre el lado izquierdo de los ejemplares, excepto cuando éstos estaban deteriorados en tal lado; el número de escamas entre la línea lateral y la aleta anal se tomó en diagonal sólo a partir de la primera escama sobre el primer radio del origen; cuando las escamas predorsales presentaron una distribución irregular no se contaron, y el número de radios simples en la aleta anal incluye los radios iniciales pequeños hasta donde fueron visibles; las escamas desde el origen de la aleta dorsal y la línea lateral fueron contadas, incluyéndose una pequeña escama al lado de la predorsal.

Las medidas se tomaron con un calibrador digital hasta centésimas de milímetro y se expresaron como porcentajes de la longitud estándar (LE) y longitud de la cabeza (LC); se utilizaron 23 caracteres morfométricos y 9 merísticos (Cuadro 1) con base en Román-Valencia (2003a). Se contó el número de vértebras, supraneurales, radios caudales procurrentes y pterigióforos de la aleta anal sobre ejemplares clareados y teñidos (C. y T.), de acuerdo con modificaciones al método descrito por Taylor y Van Dyke (1985), excepto para una población del bajo río Magdalena. Para la nomenclatura de huesos se siguió a Weitzman (1962). Los conteos de vértebras incluyen 


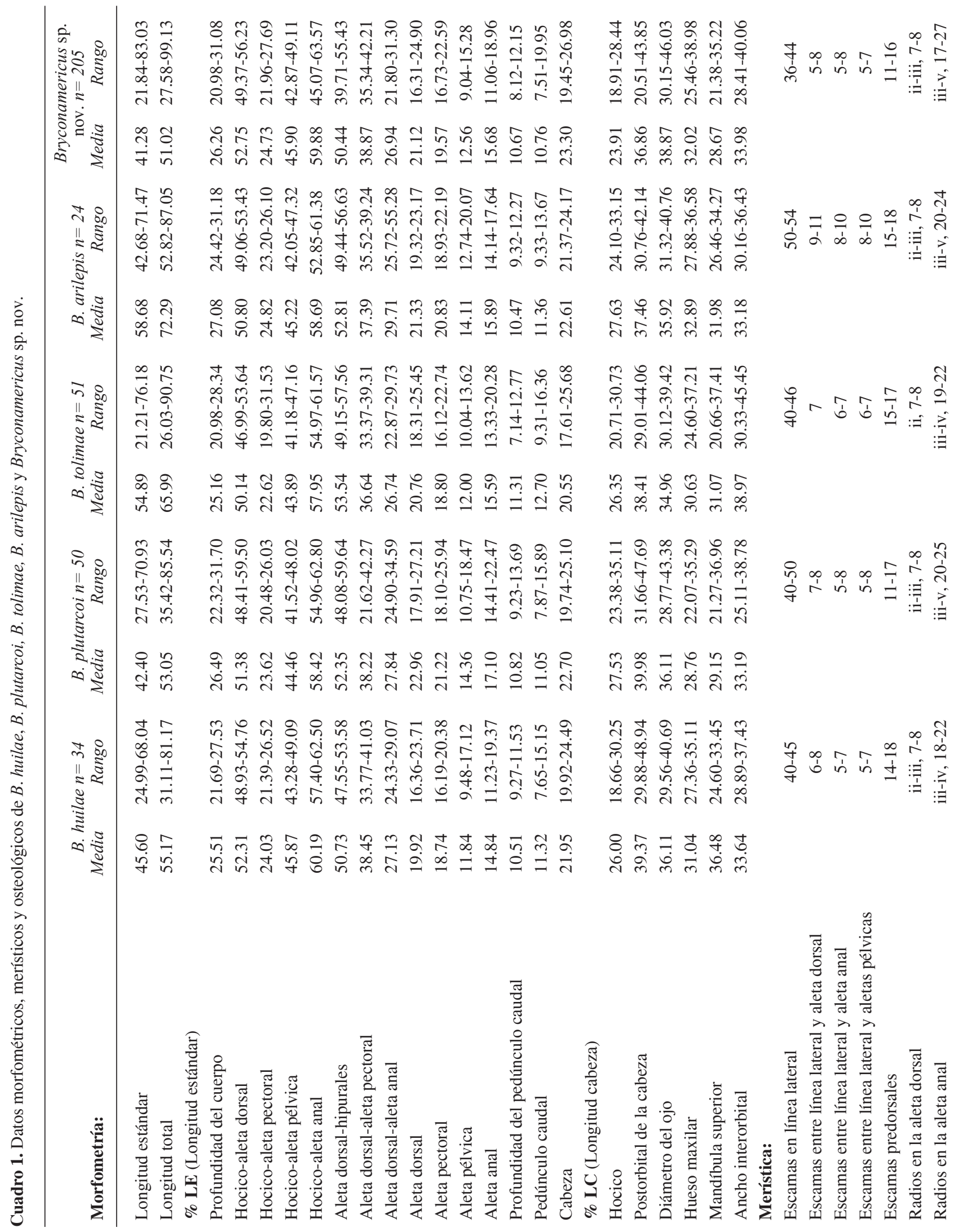




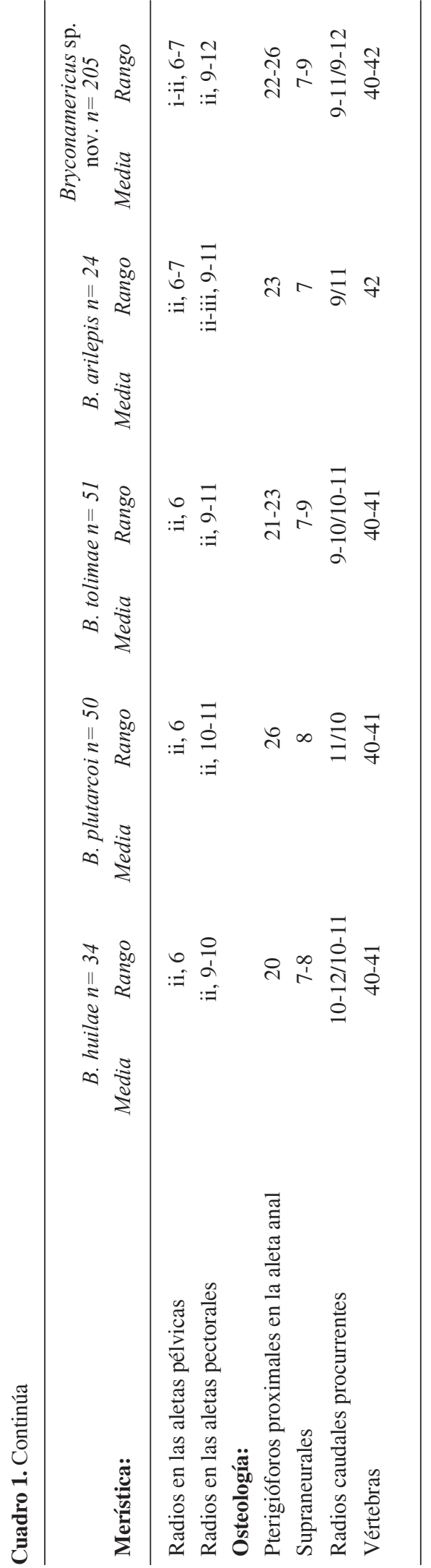

las 4 primeras del aparato de Weber. El centro terminal, los hypurales y elementos vertebrales asociados, juntos se denominan PU1+U1 y fueron contados como una sola vértebra.

Análisis de datos. La 23 medias fueron transformadas a logaritmo y se les corrigió el efecto de la talla aplicando la técnica de Burnaby (Burnaby, 1966; Rohlf y Bookstein, 1987: implementado en PAST 1,82 bajo Windows), con el fin de efectuar un análisis de componentes principales (ACP) a partir de la matriz de covarianza para evaluar: 1) la variación entre las especies registradas para el río Magdalena: B. caucanus, B. huilae, B. plutarcoi, B. tolimae, B. arilepis y Bryconamericus sp. nov., 2) la formación de grupos dentro de $B$. caucanus, y 3 ) la validez entre $H$. dentatus y $H$. decurrens vs. $B$. caucanus. Sólo se procesaron dentro del ACP los ejemplares que tuvieron una longitud estándar mayor o igual a $30 \mathrm{~mm}$, que es la talla de madurez sexual límite para B. caucanus (RománValencia y Muñoz, 2001; Román-Valencia et al., 2008c). Se construyó un índice de forma (IF) a partir del ACP que incluyó todos los datos de los taxones analizados, basado en los coeficientes de la primera componente que se obtuvieron del ACP y con base en el hecho de que la primera componente explicó el mayor porcentaje de la variabilidad del problema. Este IF se calculó usando como coeficiente la coordenada de cada medida en la primera componente y los datos de cada medida encontrada como representativa para caracterizarlas. El criterio de elección de las variables que construyeran el IF fue el que se obtuvo según lo ilustrado por el gráfico de representatividad, correlación del ACP y coeficientes mayores a 0.2. Los valores obtenidos fueron transformados a valores jerárquicos expresados de menor a mayor (con la opción rank del Sigma Plot 11,0), de donde se obtuvo un índice por ejemplar y se verificó si se presentaban tendencias de agrupación para las especies.

Para medidas y conteos con poco solapamiento o que distinguían parcialmente alguna especie de Bryconamericus se emplearon: 1), un análisis de varianza (Andeva) de un factor, para probar la hipótesis de igualdad de medias entre los taxones analizados, a partir de la variable, acompañado de una prueba de Tukey para comparar cada par de medias entre especies, cuando el Andeva mostró diferencias significativas; este análisis se efectuó también para el IF. Cuando fue necesario, por no encontrar normalidad en los datos, se aplicó un análisis de Kruskal-Wallis de un factor, junto con una prueba de comparaciones múltiples de Mann-Whitney con el valor de Bonferroni. El nivel de significancia usado para todos los análisis fue 0.05. 2), gráficos de cajas y barras con intervalos de confianza al 99 \% y desviación estándar. Las pruebas aquí aplicadas se llevaron a cabo en los programas Past 1,82, Sigma Plot 
11,0 y SPSS 9,0 todos bajo Windows.

\section{Resultados}

Las variables utilizadas se consignaron en los cuadros 1 y 2. Los caracteres merísticos que mejor distinguen a los Bryconamericus son los conteos de escamas con poros en la línea lateral, escamas predorsales y escamas entre la línea lateral y el origen de la aleta dorsal. El número de vértebras diferenció a $B$. caucanus de las otras especies registradas para el río Magdalena (37-39 vs. 40-42, Cuadros 1 y 2). Se encontró que B. arilepis se distingue de sus congéneres del río Magdalena por las escamas en la línea lateral (50-54 vs. 34-50) y escamas entre el origen de la aleta dorsal y la línea lateral (9-11 vs. 5-8).

La comparación de los Bryconamericus de la cuenca de los ríos Cauca-Magdalena y Ranchería, a través del ACP, confirmó 2 especies nuevas (Román-Valencia et al., 2008b; Román-Valencia et al., en prensa) por merística y en parte por osteología (Cuadro 1). La mayoría de los ejemplares de $B$. caucanus se ubicaron en el plano izquierdo del ACP $(33,30 \%$ de variación explicada: Cuadro 3, Fig. 1), caracterizados por la longitud del pedúnculo caudal, longitudes de las aletas anal y pélvicas (variables mejor representadas en el ACP); sin embargo, existió solapamiento con B. plutarcoi, y algunos individuos de $B$. caucanus se ubicaron afines con las demás especies distribuidas en la parte media y alta del río Magdalena. Ninguna otra especie de Bryconamericus incluida pudo distinguirse de sus congéneres afines geográficamente.

El IF construido a partir de la inclusión de las variables: longitud del hocico-origen de las aletas pélvicas, longitud del hocico-origen de la aleta anal, longitud del pedúnculo caudal, longitud de la aleta pélvica, longitud de la aleta dorsal, longitud de la aleta anal y longitud del maxilar, dilucidó especies que comparten forma similar: $B$. caucanus y B. plutarcoi, B. tolimae, B. huilae, B. arilepis y Bryconamericus sp. nov.; ambos grupos distinguibles entre sí (Cuadro 4, Fig. 2). La Kruskal-Wallis mostró diferencias significativas entre especies $(\mathrm{F}=129,9 ; \mathrm{p}=0,000)$; la prueba de Mann-Whitney confirmó que existen diferencias significativas en la forma (IF) para la mayoría de las combinaciones entre especies (Cuadro 5); B. caucanus y $B$. plutarcoi fueron las únicas que se distinguieron de las demás especies (valores de Bonferroni no corregidos y corregidos $=0,000$, Cuadro 5).

El Andeva demostró que existen diferencias significativas entre las longitudes de las aletas pélvicas entre especies de Bryconamericus $(\mathrm{F}=44,7 ; \mathrm{p}=0,000)$. La prueba de Tukey identificó que $B$. arilepis se distingue de los demás taxones $(\mathrm{Q}=7,21-13,46, \mathrm{p}=0,000-0,001) ; B$. caucanus registró diferencias significativas con $B$. huilae $(\mathrm{Q}=6,997, \mathrm{p}=0,000), B$. arilepis $(\mathrm{Q}=5,548, \mathrm{p}=0,001) \mathrm{y}$ Bryconamericus sp. nov. $(\mathrm{Q}=7,911, \mathrm{p}=0,000$ : Cuadro 6). También se observaron discrepancias en las longitudes de la aleta anal al confrontar las especies de Bryconamericus analizadas $(\mathrm{F}=44,03 ; \mathrm{p}=0,000)$. La prueba de Tukey identificó contrastes entre Bryconamericus sp. nov., $B$. caucanus, B. arilepis, B. tolimae y B. huilae con al menos 3 de los demás taxones (Cuadro 6).

La prueba Kruskal-Wallis fue significativa para el número de escamas con poros en la línea lateral $(\mathrm{H}=$ 524,$8 ; \mathrm{Hc}=532,8 ; \mathrm{p}=0,000$ ), las escamas predorsales $(\mathrm{H}=307,3 ; \mathrm{Hc}=323,3 ; \mathrm{p}=0,000)$ y las escamas entre la línea lateral y el origen de la aleta dorsal $(\mathrm{H}=328,7$; $\mathrm{Hc}=367,0 ; \mathrm{p}=0,000)$, además, la comparación de Mann-Whitney indicó diferencias para cada una de las especies de Bryconamericus (excepto B. tolimae vs. B. plutarcoi), al contrastarlas con las demás especies (Cuadro 7); el gráfico con intervalos de confianza al $99 \%$ sugirió resultados similares a los obtenidos por la prueba MannWhitney (Fig. 3). Sin embargo, otros análisis estadísticos concentrados en detectar solapamiento en estos conteos entre los Bryconamericus de la cuenca de los ríos CaucaMagdalena y Ranchería demuestran que B. caucanus puede diferenciarse de los otros taxones en las escamas con poros en la línea lateral (excepto de Bryconamericus sp. nov.); $B$. arilepis puede distinguirse de los demás Bryconamericus en el conteo de escamas con poros en la línea lateral y escamas entre la línea lateral y el origen de la aleta dorsal, debido en cierta medida a que la mayoría de los individuos examinados presentaron registros altos en relación con las demás especies (Cuadro 8); los gráficos de cajas confirmaron, en parte, estas tendencias en $B$. caucanus y B. arilepis (Fig. 4).

Para el análisis de la variación poblacional de $B$. caucanus el ACP confirmó una sola forma presente en el río Cauca-Magdalena y Ranchería (Cuadro 3, Fig. 5), corroborado por el conteo de vértebras y espinas en los machos distribuidas en los radios de la aleta anal y pélvica. Las poblaciones de B. caucanus de la parte baja del río Cauca son las que poseen mayor afinidad en forma con las demás poblaciones del Ranchería, alto y medio Cauca y bajo Magdalena; ninguna población de B. caucanus es lo suficientemente distinta del resto como para formar una agrupación aislada en el ACP (Fig. 5: variación explicada por la primera y segunda componente igual a 35,98 \%). Las longitudes de las aletas pélvicas, pedúnculo caudal, postorbital de la cabeza y hocico fueron las variables con mejor representación en el ACP y de mayor discriminación (Fig. 5). La merística y osteología no demostró tampoco ninguna diferencia entre poblaciones (Cuadro 2). En las poblaciones de la cuenca alta del río Cauca se encontraron 


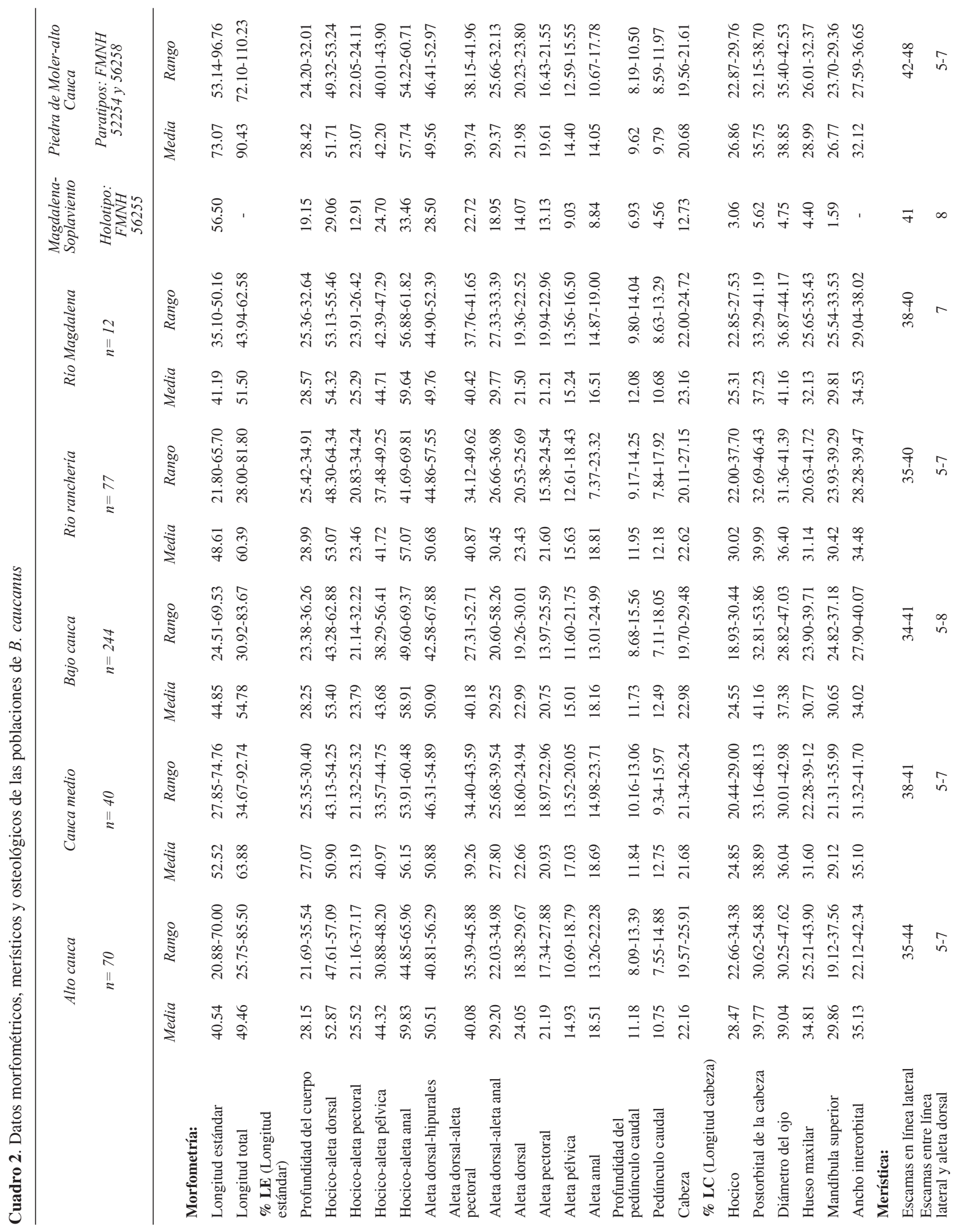




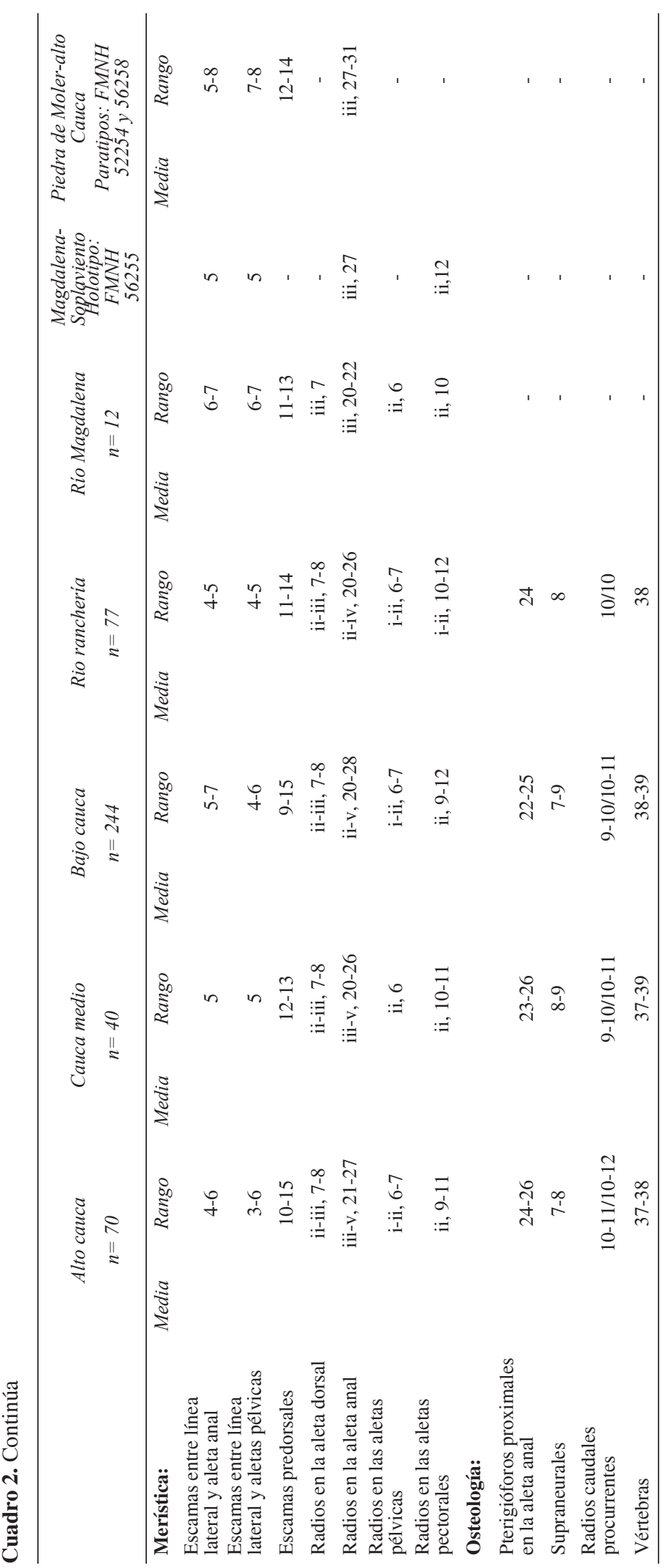

individuos con conteos de escamas con poros en la línea lateral y radios en la aleta anal ligeramente superiores al resto de las poblaciones (Cuadro 2: alto Cauca); sin embargo, algunos fueron valorados dentro de la comparación con $H$. decurrens y $H$. dentatus o tomados como valores atípicos, dado el amplio número de individuos analizados (mayor a 400) y dentro de las gráficas estadísticas entre especies no fueron incluidos (Figs. 3 y 4) pero si registrados en los cuadros (Cuadro 2).

En cuanto a la validez de $H$. decurrens, $H$. dentatus y B. caucanus el ACP demostró que es lo mismo en forma (Fig. 6: variación explicada por la primera y segunda componentes igual a 30,85 $\%$; Cuadro 3). El conteo de escamas con poros en la línea lateral y radios en la aleta anal en $H$. decurrens y $H$. dentatus son ligeramente más altos a los rangos de B. caucanus, pero considerados dentro de la variación de la especie.

\section{Discusión}

El índice de forma permitió caracterizar a B. caucanus y B. plutarcoi como las especies más afines en forma, pero no idénticas, dentro la cuenca de los ríos Cauca-Magdalena y Ranchería, y a su vez distinguibles de $B$. huilae, $B$. tolimae, $B$. arilepis y Bryconamericus sp. nov.; no obstantela amplia variación de las poblaciones de $B$. caucanus representada por la dispersión de los datos morfométricos en el ACP, el número de vértebras (37-39) y escamas con poros en la línea lateral (34-44) son caracteres taxonómicos útiles para distinguir $B$. cancanus de las demás especies de Bryconamericus del río Magdalena, excepto en el último carácter, de Bryconamericus sp. nov. (36-44 escamas con poros en la línea lateral). Los Bryconamericus de la cuenca de los ríos Cauca-Magdalena y Ranchería están en alopatría, encontrándose B. caucanus únicamente en la cuenca de los ríos Cauca, bajo Magdalena, Sinú y Ranchería, mientras las demás especies se distribuyen en la cuenca media y alta del río Magdalena (Fig. 7).

Las longitudes de la aleta anal y pélvica son útiles como caracteres taxonómicos. González-Díaz et al. (2005) estudiaron la variación poblacional de un complejo taxonómico dentro de Profundulidae y encontraron que los caracteres morfológicos de mayor poder discriminatorio en el ámbito de especie y poblaciones han sido de relevancia para estudios filogenéticos de esta familia. Sin embargo, en 


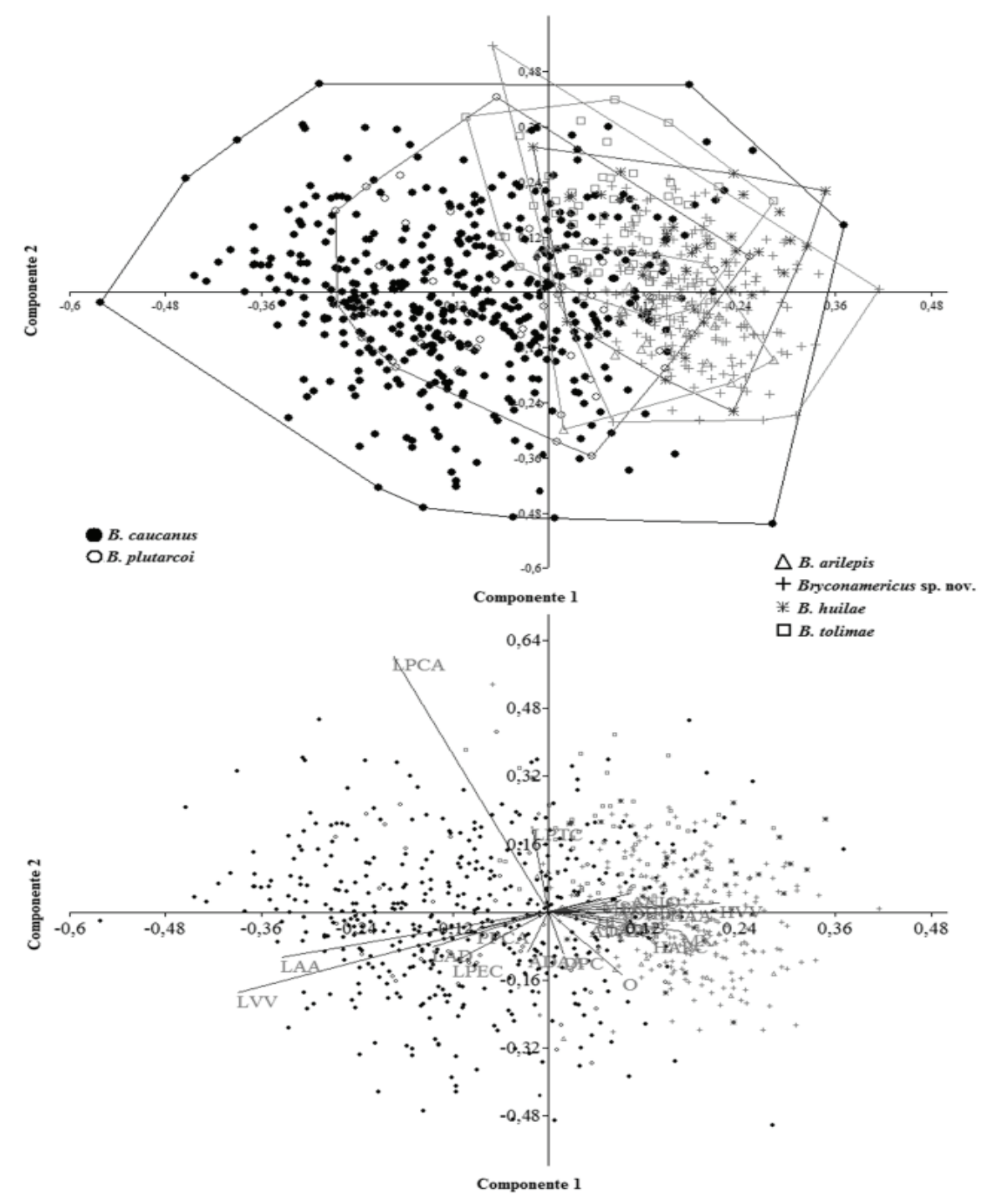

Figura 1. Análisis de Componentes Principales (ACP) para las especies de Bryconamericus de la cuenca del río Cauca-Magdalena y Ranchería. Entre la primera y segunda componente se explicó el $33,30 \%$ de la variación. DCP: profundidad del cuerpo; LC: longitud de la cabeza; HAP: longitud del hocico-origen de las aletas pectorales; O: diámetro horizontal del ojo; IO: ancho interorbital; PPC: profundidad del pedúnculo caudal; $\mathrm{H}$ : longitud del hocico; HVV: distancia hocicoorigen de las aletas pélvicas; HAA: distancia hocico-origen de la aleta anal; Msup: longitud de la mandíbula superior; LAD: longitud de la aleta dorsal; LAA: longitud de la aleta anal; ADAP : distancia del origen de la aleta dorsal-origen de las aletas pectorales; LPC: longitud de las aletas pectorales; LAVLAPEL: longitud de las aletas pélvicas; LS: longitud estándar; LPCA: longitud del pedúnculo caudal; ADHIP: distancia del origen aleta dorsal-base de los hipurales; LPTC: longitud postorbital; LT: longitud total; ADAA: distancia del origen de la aleta dorsal-origen de la aleta anal; MX: longitud del hueso maxilar.

caucanus de la parte alta, media y baja del río Cauca, bajo Magdalena y Ranchería no pueden diferenciarse por forma a lo obtenido por el ACP, a osteología y merística. Además, es evidente el solapamiento entre algunos ejemplares, al menos en forma, de las poblaciones de B. caucanus con B. huilae,

nuestro caso las características morfológicas y merísticas han sido valoradas para un contexto filogenético pero han fallado la prueba de congruencia como sinapomorfías (Serra y Langeani, 2001; 2006; Vanegas-Ríos, 2008).

Bryconamericus caucanus es una especie de amplia distribución en Colombia, localizada en toda la cuenca del río Cauca, bajo Magdalena, ríos Sinú y Ranchería (Fig. 7). Se corrobora y complementan sus registros (RománValencia, 2003a) y se invalidan las citas en Eigenmann y Allen (1942), Miles (1947), (Tortonose 1941, 1942 en Böhlke, 1958), Fowler (1951), Ortega y Vari (1986) y Barriga (1991). Las poblaciones analizadas de $B$.
B. tolimae, B. plutarcoi, B. arilepis y Bryconamericus sp. nov. Dentro de las especies de Bryconamericus, $B$. emperador es una especie que también se caracteriza por su amplia distribución en el Pacífico colombiano y oriente de Panamá en América Central (Román-Valencia, 2002, 2003a; Reeves y Bermingham, 2006; Vanegas-Ríos, 2008. De igual manera, para $B$. alpha y $B$. cismontanus en la Orinoquia (Román-Valencia, 2005). Román-Valencia y Muñoz (2001) afirmaron que B. caucanus no efectúa migraciones; sin embargo, para el modelo actual de distribución geográfica de $B$. caucanus podría considerarse tentativamente un modelo metapoblacional. 


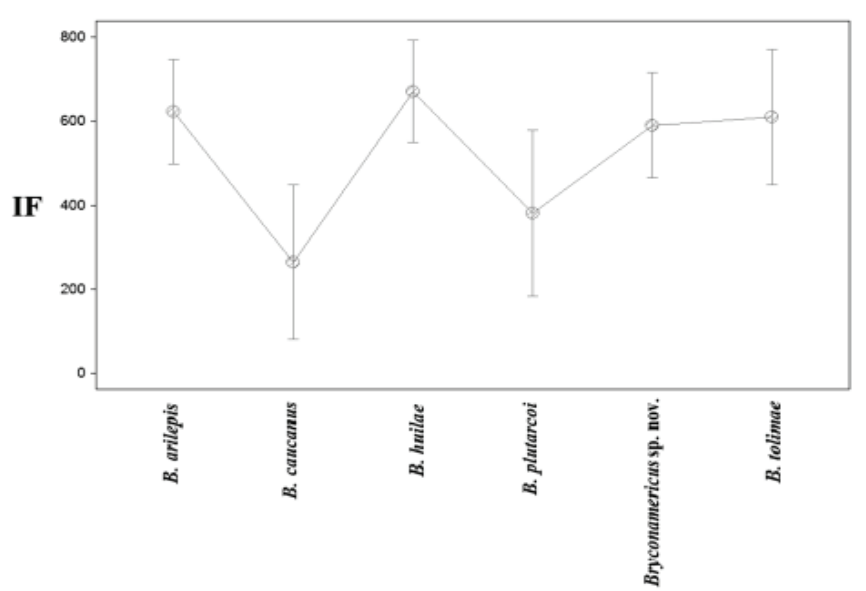

Figura 2. Variación del índice de forma (IF) entre las especies de Bryconamericus de la cuenca de los ríos Cauca-Magdalena y Ranchería. Círculos representan la media, los intervalos la desviación estándar.
Los registros de Bryconamericus sp. en Jiménez et al. (1998) son, con base en nuestros resultados, B. caucanus. Además, la ausencia de Bryconamericus en el listado de Mojica et al. (2006) para el río Ranchería es un error, se infiere que no efectuaron un examen exhaustivo. Maldonado-Ocampo et al. (2005) registraron B. caucanus para el río Anchique (cuenca del Magdalena); sin embargo, este registro se tomó de un estudio faunístico para el río Prado y Amoyá, y no de una revisión comparada con material (por ejemplo, con B. tolimae); por tal motivo se considera poco confiable.

Bryconamericus caucanus también puede distinguirse de las demás especies analizadas por el dimorfismo sexual secundario de los machos, representado en ganchos o espinas en las aletas anal y pélvicas, donde los radios de la aleta anal tienen espinas a partir del primero más largo y simple hasta el séptimo u décimo ramificado y se localizan alrededor de la parte media y superior del radio (RománValencia et al., en prensa). Mientras B. tolimae, B. huilae y Bryconamericus sp. nov. poseen espinas sobre los radios a

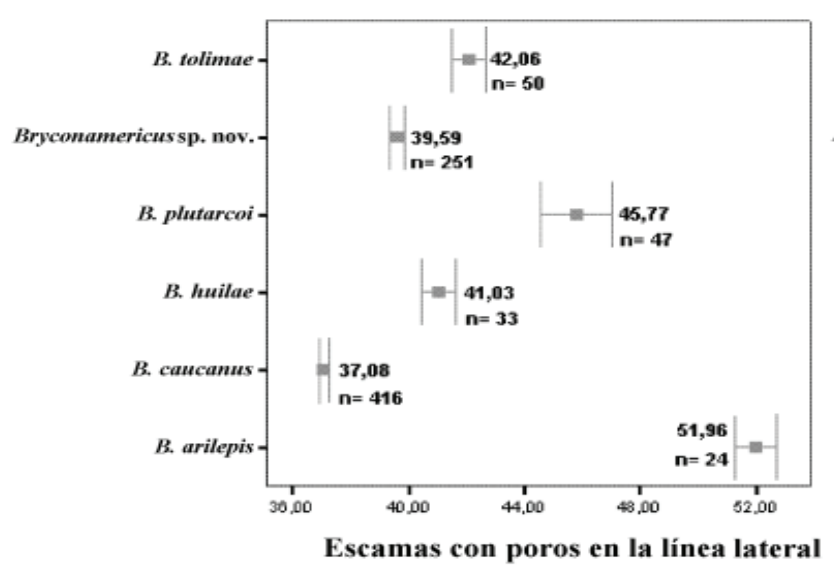

b

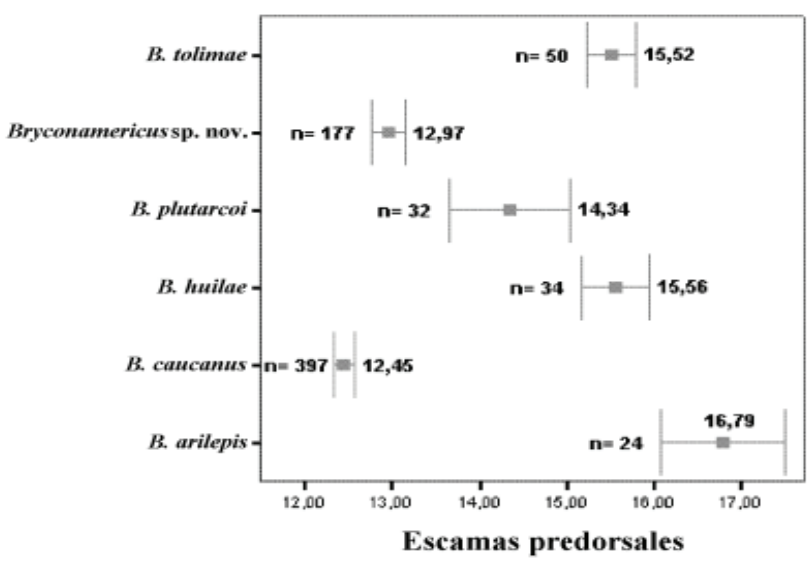

c

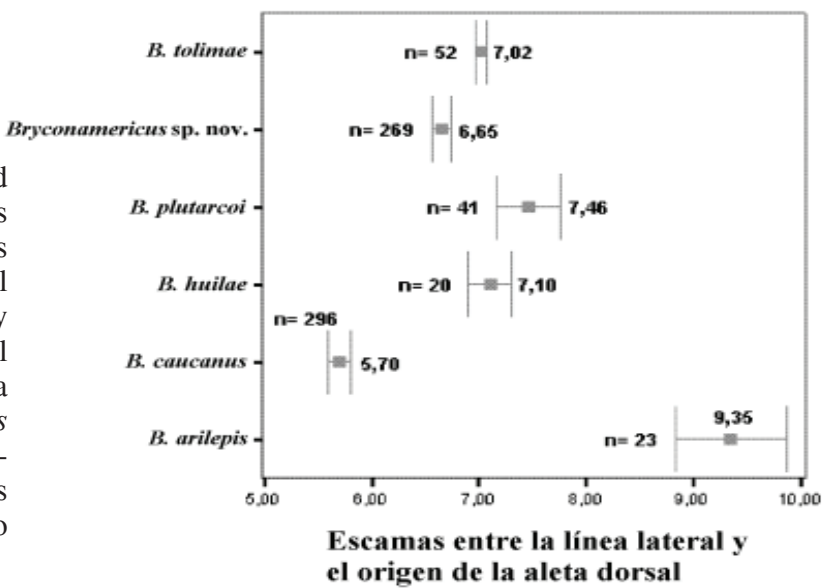

Figura 3. Media con probabilidad al $99 \%$ de confianza (líneas verticales) del número de escamas con poros en la línea lateral (a), escamas predorsales (b), y escamas entre la línea lateral y el origen de la aleta dorsal (c), para las especies de Bryconamericus de la cuenca de los ríos CaucaMagdalena y Ranchería. Los números cercanos al cuadro representan la media aritmética.

el origen de la aleta dorsal 
a

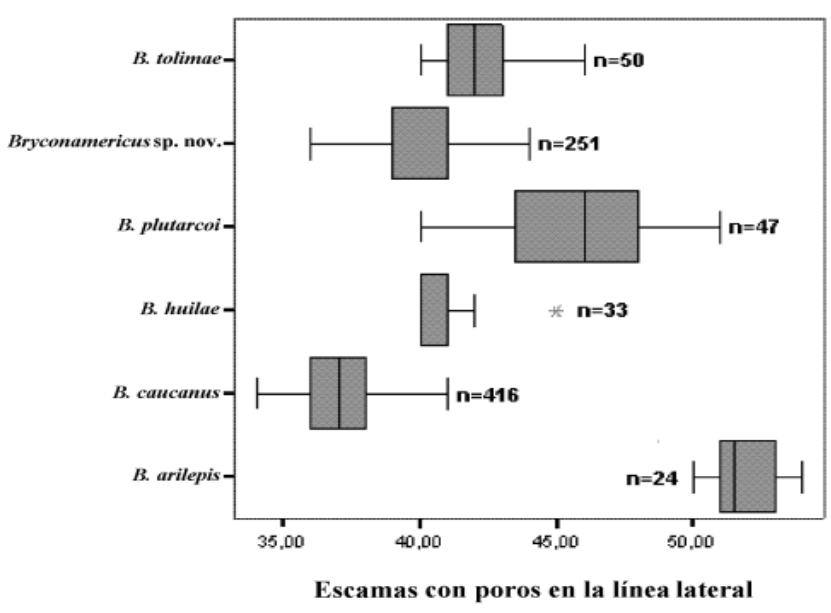

c b

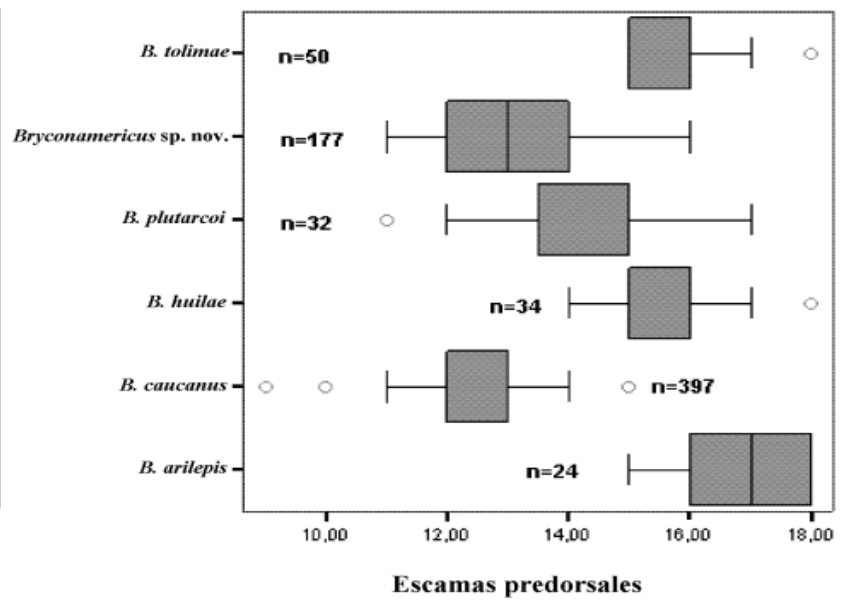

Figura 4. Variación del conteo de escamas con poros en la línea lateral (a), escamas predorsales (b), y escamas entre la línea lateral y el origen de la aleta dorsal (c), para las especies de Bryconamericus de la cuenca de los ríos Cauca-Magdalena y Ranchería. Los asteriscos representan valores atípicos.

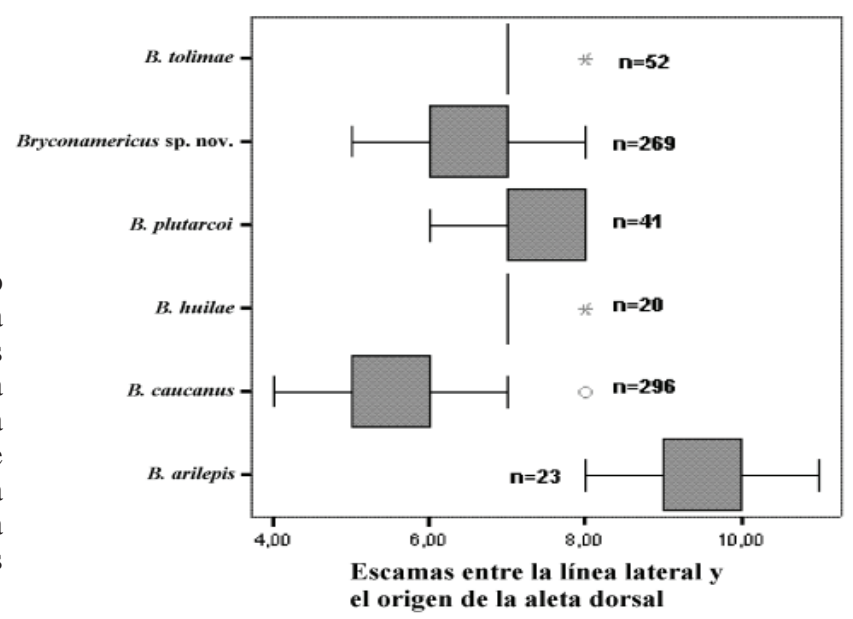

de las aletas anal, pélvicas, pectorales y dorsal (VanegasRíos, 2008; Román-Valencia et al., 2008b; RománValencia et al., En prensa).

El ACP plantea que $H$. decurrens y $H$. dentatus no se distinguen de $B$. caucanus. Sin embargo, el número de radios ramificados en la aleta anal registra variación: H. dentatus 28-31, H. decurrens 27 y B. caucanus 20-28 (véase también Román-Valencia, 2003a). Una situación similar acontece con el número de escamas en la línea lateral: $H$. dentatus $42-48, H$. decurrens 41 y $B$. caucanus 34-44 (34-46 según Román-Valencia, 2003a). Además, $H$. dentatus se describe para Piedra Moler, La Paila en alto río Cauca, como un Bryconamericus (Eigenmann, 1913). En ningún caso Eigenmann $(1922,1927)$ incluye esta especie en el género Hemibrycon Günther 1864. Él también suministra una breve descripción con los mismos caracteres publicados en 1913 para la especie $H$. dentatus y la registra para Villavicencio en alto Meta (Orinoquia). Evidentemente debe ser H. metae, como lo anotaron Myers (1930), Román-Valencia y Ruiz-C. (2007) y Román-Valencia y Arcila-Mesa (2008). Esta especie, Miles (1947), Dahl (1971) y Géry (1962, 1972, 1977) la listan como $H$. dentatus, pero Géry (1962) anota que está estrechamente relacionada con Bryconamericus. Al examinar los dientes del maxilar observamos de 3 a 9, diagnóstico para Bryconamericus. Así también, al inspeccionar las ilustraciones del maxilar en $H$. dentatus y B. caucanus en Eigenmann (1922) observamos que para ambos peces la parte de este hueso con dientes es más corta que el segmento sin dientes. Hemibrycon dentatus nunca ha sido colectada por el primer autor (C. R.-V.) en río La Vieja en Piedra Moler, ni en alguna otra localidad del alto Cauca (Román-Valencia 1993, 1995, 2001; RománValencia y Muñoz, 2001). También, al comparar los datos de Román-Valencia (2003a, tabla 1) para B. caucanus con el material tipo de $H$. dentatus, no se observan diferencias. En los tipos de $H$. dentatus se encontró dimorfismo sexual secundario representado por espinas en las aletas anal y 


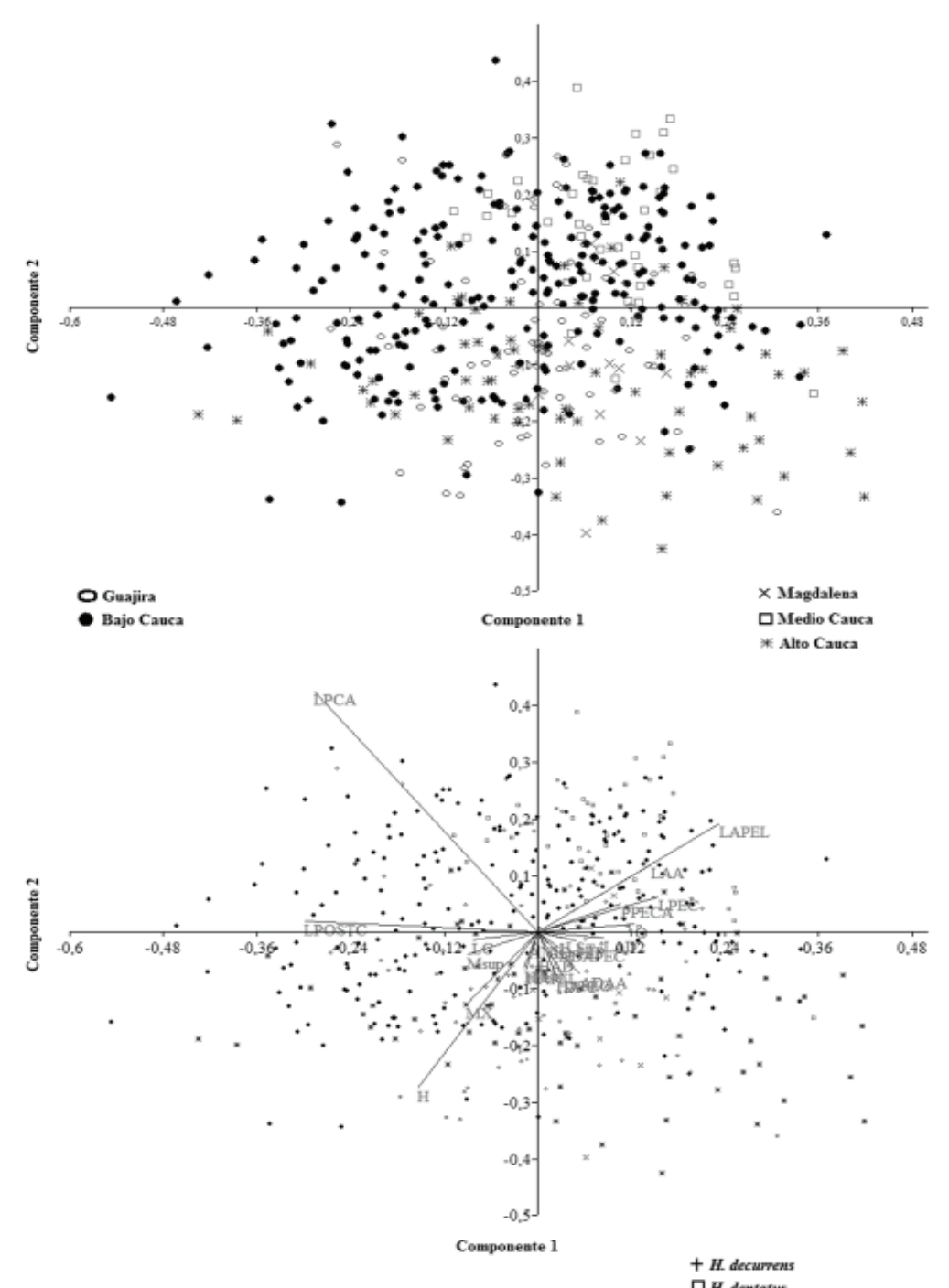

Figura 5. Diagrama de la primera y la segunda componente de las poblaciones de B. caucanus (puntos). Entre la primera y segunda componente se explicó el 35,98 \% de la variación. DCP: profundidad del cuerpo; LC: longitud de la cabeza; HAP: longitud del hocico-origen de las aletas pectorales; O: diámetro horizontal del ojo; IO: ancho interorbital; PPC: profundidad del pedúnculo caudal; $\mathrm{H}$ : longitud del hocico; $\mathrm{HVV}$ : distancia hocico-origen de las aletas pélvicas; HAA: distancia hocico-origen de la aleta anal; Msup: longitud de la mandíbula superior; LAD: longitud de la aleta dorsal; LAA: longitud de la aleta anal; ADAP : distancia del origen de la aleta dorsal-origen de las aletas pectorales; LPC: longitud de las aletas pectorales; LAV-LAPEL: longitud de las aletas pélvicas; LS: longitud estándar; LPCA: longitud del pedúnculo caudal; ADHIP: distancia del origen aleta dorsal-base de los hipurales; LPTC: longitud postorbital; LT: longitud total; ADAA: distancia del origen de la aleta dorsal-origen de la aleta anal; MX: longitud del hueso maxilar.

Figura 6. Variación poblacional de $B$. caucanus vs. H. decurrens (holotipo FMNH 56255) y $H$. dentatus (paratipos FMNH 56252, 56254). Entre la primera y segunda componente se explicó el 30,85\% de la variación.

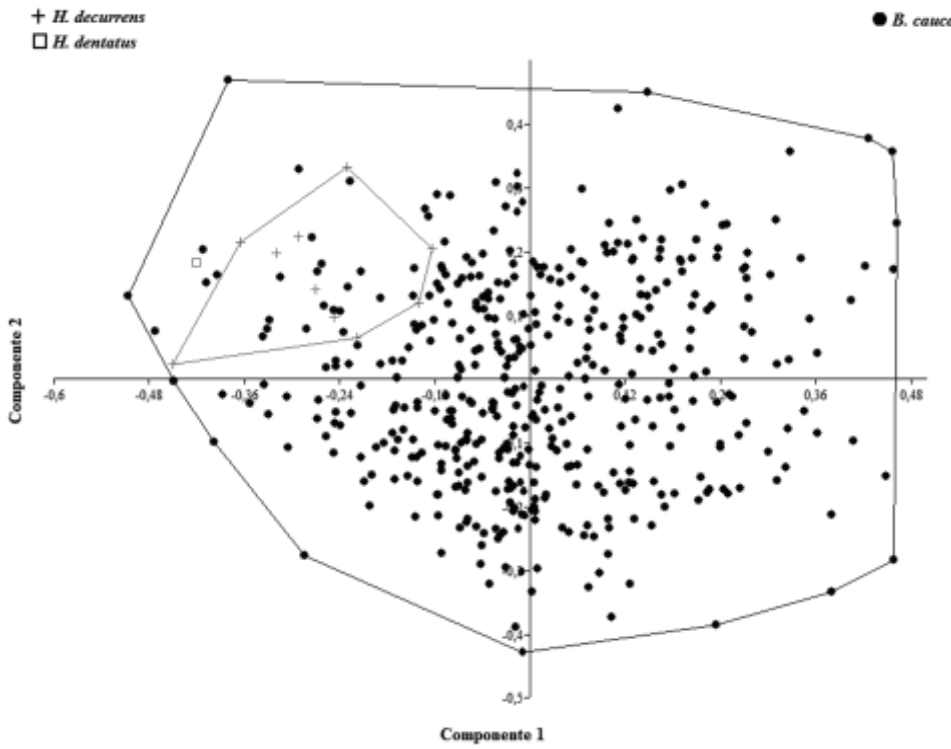

pélvicas en machos, lo que coincide con $B$. caucanus. Por lo tanto, $H$. dentatus se considera sinónimo moderno de B. caucanus. Géry (1966) puntualiza que algunas especies de Hemibrycon (H. boquiae, $H$. decurrens y $H$. dentatus) poseen un bajo número de dientes en el maxilar. Esta observación en relación con los pocos dientes en $H$. boquiae, $H$. decurrens y $H$. dentatus corresponde a $B$. caucanus, y en el caso de $H$. boquiae, porque ambas especies coexisten en la quebrada Boquia afluente del río Quindío, drenaje del alto Cauca (Román-Valencia et al., 2008c). Eigenmann (1913) inadvertidamente incluyo ejemplares de $B$. caucanus en la descripción de H. boquiae. 
Cuadro 3. Valores propios (eigenvalue) de los ACP: 1 , comparación entre las especies de Bryconamericus de la cuenca de los ríos Cauca-Magdalena y Ranchería; 2, comparación entre las poblaciones de $B$. caucanus; 3 , comparación entre $B$. caucanus, $H$. decurrens y $H$. dentatus

\begin{tabular}{|c|c|c|c|}
\hline & & $A C P$ & \\
\hline Componente & 1 & 2 & 3 \\
\hline 1 & 0.0302 & 0.028 & 0.0316 \\
\hline 2 & 0.0243 & 0.024 & 0.0263 \\
\hline 3 & 0.0153 & 0.014 & 0.0156 \\
\hline 4 & 0.0128 & 0.013 & 0.0146 \\
\hline 5 & 0.0110 & 0.010 & 0.0130 \\
\hline 6 & 0.0083 & 0.007 & 0.0106 \\
\hline 7 & 0.0080 & 0.007 & 0.0098 \\
\hline 8 & 0.0074 & 0.007 & 0.0085 \\
\hline 9 & 0.0064 & 0.006 & 0.0080 \\
\hline 10 & 0.0061 & 0.004 & 0.0072 \\
\hline 11 & 0.0051 & 0.004 & 0.0063 \\
\hline 12 & 0.0045 & 0.004 & 0.0055 \\
\hline 13 & 0.0041 & 0.003 & 0.0053 \\
\hline 14 & 0.0039 & 0.003 & 0.0050 \\
\hline 15 & 0.0034 & 0.003 & 0.0044 \\
\hline 16 & 0.0029 & 0.002 & 0.0038 \\
\hline 17 & 0.0028 & 0.002 & 0.0032 \\
\hline 18 & 0.0021 & 0.001 & 0.0027 \\
\hline 19 & 0.0019 & 0.001 & 0.0024 \\
\hline 20 & 0.0013 & 0.001 & 0.0016 \\
\hline 21 & 0.0012 & 0.001 & 0.0014 \\
\hline 22 & 0.0006 & 0.001 & 0.0009 \\
\hline 23 & 0.0000 & 0.000 & 0.0000 \\
\hline
\end{tabular}

Hemibrycon decurrens se describe como un Bryconamericus del bajo Magdalena, en el sector del Canal del Dique (Eigenmann, 1913). Sin embargo, Eigenmann (1922, 1927) la incluye en el género Hemibrycon y suministra una breve descripción, con iguales caracteres a los que usó en 1913 para la especie $H$. decurrens. Miles (1947), Dahl (1971) y Géry $(1962,1977)$ la ubican como H. decurrens. Reconocen como el carácter principal para definir el género Hemibrycon, la presencia de dientes a lo largo de todo el borde del maxilar. Tanto en Eigenmann $(1913,1927)$ como en Géry (1962) la parte del maxilar con dientes es menor que el segmento sin dientes, esto es, con pocos dientes (6 a 9) que no cubren el borde del
Cuadro 4. Indice de forma (IF) para el ACP efectuado para los datos de todas las especies de Bryconamericus de la cuenca del río Cauca-Magdalena y Ranchería. MAX = valor máximo, $\mathrm{MIN}=$ valor mínimo, VAR = varianza

\begin{tabular}{lcccc}
\hline & Media & Min & Max & Var \\
\hline $\begin{array}{l}\text { B. caucanus } \\
\text { Bryconamericus }\end{array}$ & 264.12 & 1.00 & 798.00 & 33791.98 \\
$\begin{array}{l}\text { sp. nov. } \\
\text { B. arilepis }\end{array}$ & 590.15 & 241.00 & 806.00 & 15642.38 \\
B. plutarcoi & 622.58 & 367.00 & 801.00 & 15438.60 \\
B. tolimae & 610.45 & 282.00 & 797.00 & 25974.93 \\
B. huilae & 670.18 & 292.00 & 805.00 & 14816.78 \\
\hline
\end{tabular}

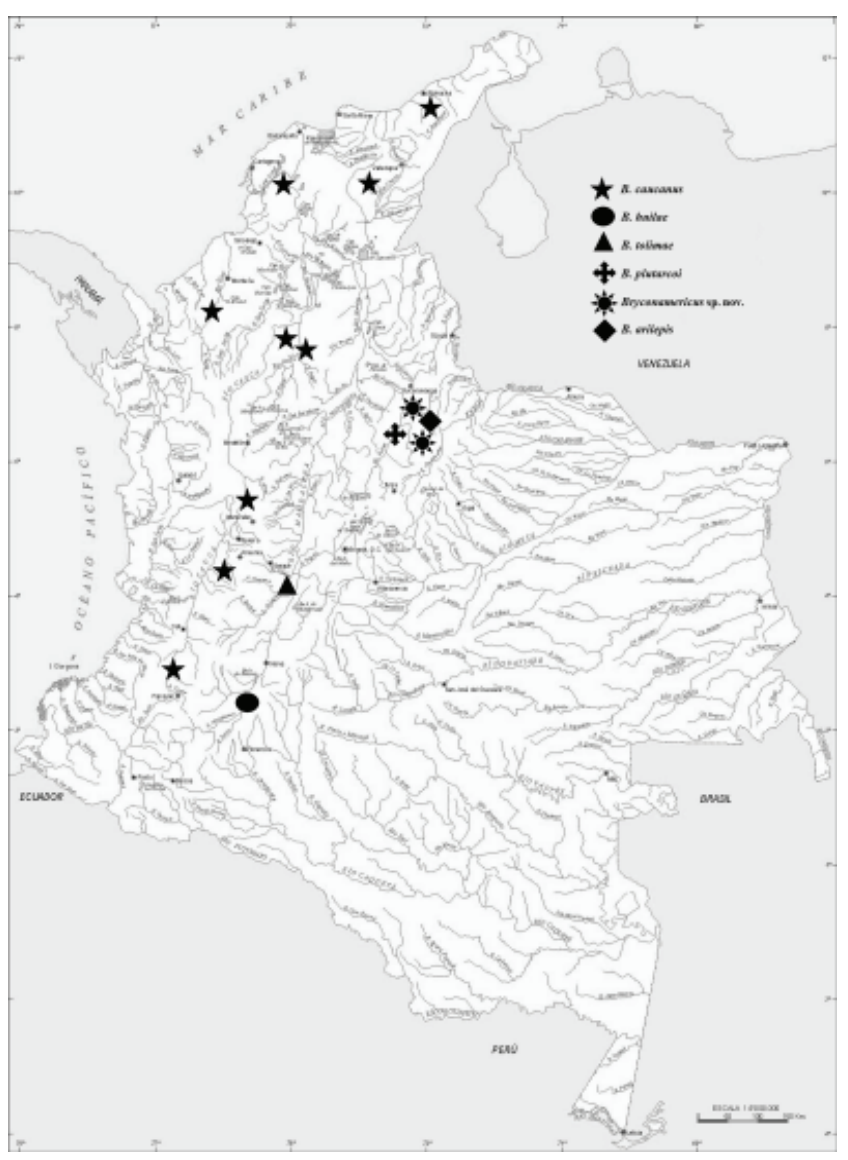

Figura 7. Distribución geográfica de las especies del género Bryconamericus para la cuenca de los ríos Cauca-Magdalena, Sinú y Ranchería. No se presentan localidades exhaustivas.

maxilar, carácter que es diagnóstico para Bryconamericus. En estas condiciones, es mejor ubicar la especie en el género Bryconamericus siguiendo a Eigenmann (1913), y como B. caucanus con base en los resultados de nuestro 
Cuadro 5. Prueba de Mann-Whitney para la comparación del índice de forma entre especies de Bryconamericus de la cuenca del río Cauca-Magdalena y Ranchería. La parte superior de la matriz muestra los valores de Bonferroni no corregidos y la inferior los corregidos. Significativos $<0.05$

\begin{tabular}{lcccccc}
\hline & B. caucanus & Bryconamericus sp. nov. & B. tolimae & B. plutarcoi & B. huilae & B. arilepis \\
\hline B. caucanus & $*$ & 0.000 & 0.000 & 0.000 & 0.000 & 0.000 \\
Bryconamericus sp. nov. & 0.000 & $*$ & 0.107 & 0.000 & 0.000 & 0.213 \\
B. tolimae & 0.000 & 1.603 & $*$ & 0.000 & 0.101 & 0.932 \\
B. plutarcoi & 0.001 & 0.000 & 0.000 & $*$ & 0.000 & 0.000 \\
B. huilae & 0.000 & 0.004 & 1.517 & 0.000 & $*$ & 0.094 \\
B. arilepis & 0.000 & 3.194 & 13.980 & 0.000 & 1.415 & $*$ \\
\hline
\end{tabular}

Cuadro 6. Prueba de Tukey para la comparación de longitud de la aleta pélvica y longitud de la aleta anal (variables con los coeficientes más altos en la primera componente), entre especies de Bryconamericus de la cuenca del río Cauca-Magdalena y Ranchería. La parte superior de la matriz muestra los valores de p y la inferior los del estadístico Q. Significativos $<0.05$

\begin{tabular}{|c|c|c|c|c|c|c|}
\hline \multicolumn{2}{|c|}{ B. caucanus } & Bryconamericus sp. nov. & B. tolimae & B. plutarcoi & B. huilae & B. arilepis \\
\hline \multicolumn{7}{|c|}{ Longitud de la aleta pélvica } \\
\hline B. caucanus & $*$ & 0.000 & 0.848 & 0.055 & 0.000 & 0.001 \\
\hline Bryconamericus sp. nov. & 7.911 & $*$ & 0.000 & 0.061 & 0.987 & 0.000 \\
\hline B. tolimae & 1.665 & 6.246 & * & 0.573 & 0.002 & 0.000 \\
\hline B. plutarcoi & 3.983 & 3.929 & 2.317 & $*$ & 0.271 & 0.000 \\
\hline B. huilae & 6.997 & 0.914 & 5.331 & 3.014 & $*$ & 0.000 \\
\hline B. arilepis & 5.548 & 13.460 & 7.213 & 9.530 & 12.540 & * \\
\hline \multicolumn{7}{|c|}{ Longitud de la aleta anal } \\
\hline B. caucanus & $*$ & 0.000 & 0.999 & 0.010 & 0.000 & 0.061 \\
\hline Bryconamericus sp. nov. & 7.921 & $*$ & 0.000 & 0.225 & 0.977 & 0.000 \\
\hline B. tolimae & 0.490 & 8.411 & * & 0.003 & 0.000 & 0.146 \\
\hline B. plutarcoi & 4.770 & 3.151 & 5.260 & $*$ & 0.674 & 0.000 \\
\hline B. huilae & 6.870 & 1.051 & 7.360 & 2.100 & $*$ & 0.000 \\
\hline B. arilepis & 3.927 & 11.850 & 3.438 & 8.697 & 10.800 & $*$ \\
\hline
\end{tabular}

Cuadro 7. Prueba de Mann-Whitney para la comparación del conteo de: escamas con poros en la línea lateral, escamas predorsales y escamas entre la línea lateral y el origen de la aleta dorsal entre cada especie de Bryconamericus de la cuenca de los ríos CaucaMagdalena y Ranchería. La parte superior de la matriz muestra los valores de Bonferroni no corregidos y la inferior los corregidos. Significativos $<0.05$

\begin{tabular}{lcccccc}
\hline & B. caucanus & B. arilepis & Bryconamericus sp. nov. & B. huilae & B. plutarcoi & B. tolimae \\
\hline & & \multicolumn{5}{c}{ Escamas por poros en la línea lateral } \\
B. caucanus & $*$ & 0.000 & 0.000 & 0.000 & 0.000 & 0.000 \\
B. arilepis & 0.000 & $*$ & 0.000 & 0.000 & 0.000 & 0.000 \\
Bryconamericus sp. nov. & 0.000 & 0.000 & $*$ & 0.000 & 0.000 & 0.000 \\
B. huilae & 0.000 & 0.000 & 0.000 & $*$ & 0.000 & 0.001 \\
B. plutarcoi & 0.000 & 0.000 & 0.000 & 0.000 & $*$ & 0.000 \\
B. tolimae & 0.000 & 0.000 & 0.000 & 0.009 & 0.000 & $*$
\end{tabular}


Cuadro 7. Continúa

\begin{tabular}{lcccccc}
\hline & \multicolumn{7}{c}{ Escamas predorsales } \\
B. caucanus & $*$ & 0.000 & 0.000 & 0.000 & 0.000 & 0.000 \\
B. arilepis & 0.000 & $*$ & 0.000 & 0.001 & 0.000 & 0.000 \\
Bryconamericus sp. nov. & 0.000 & 0.000 & $*$ & 0.000 & 0.000 & 0.000 \\
B. huilae & 0.000 & 0.014 & 0.000 & $*$ & 0.000 & 0.785 \\
B. plutarcoi & 0.000 & 0.000 & 0.000 & 0.006 & $*$ & 0.000 \\
B. tolimae & 0.000 & 0.003 & 0.000 & 11.770 & 0.003 & $*$ \\
& \multicolumn{7}{c}{ Escamas en la línea lateral y el origen de la aleta dorsal } \\
B. caucanus & $*$ & 0.000 & 0.000 & 0.000 & 0.000 & 0.000 \\
B. arilepis & 0.000 & $*$ & 0.000 & 0.000 & 0.000 & 0.000 \\
Bryconamericus sp. nov. & 0.000 & 0.000 & $*$ & 0.005 & 0.000 & 0.000 \\
B. huilae & 0.000 & 0.000 & 0.078 & $*$ & 0.018 & 0.602 \\
B. plutarcoi & 0.000 & 0.000 & 0.000 & 0.275 & $*$ & 0.000 \\
B. tolimae & 0.000 & 0.000 & 0.004 & 9.028 & 0.004 & $*$ \\
\hline
\end{tabular}

Cuadro 8. Frecuencias absolutas del conteo de escamas con poros en la línea lateral, escamas predorsales y escamas entre la línea lateral y el origen de la aleta dorsal. La letra n representa el tamaño de muestra

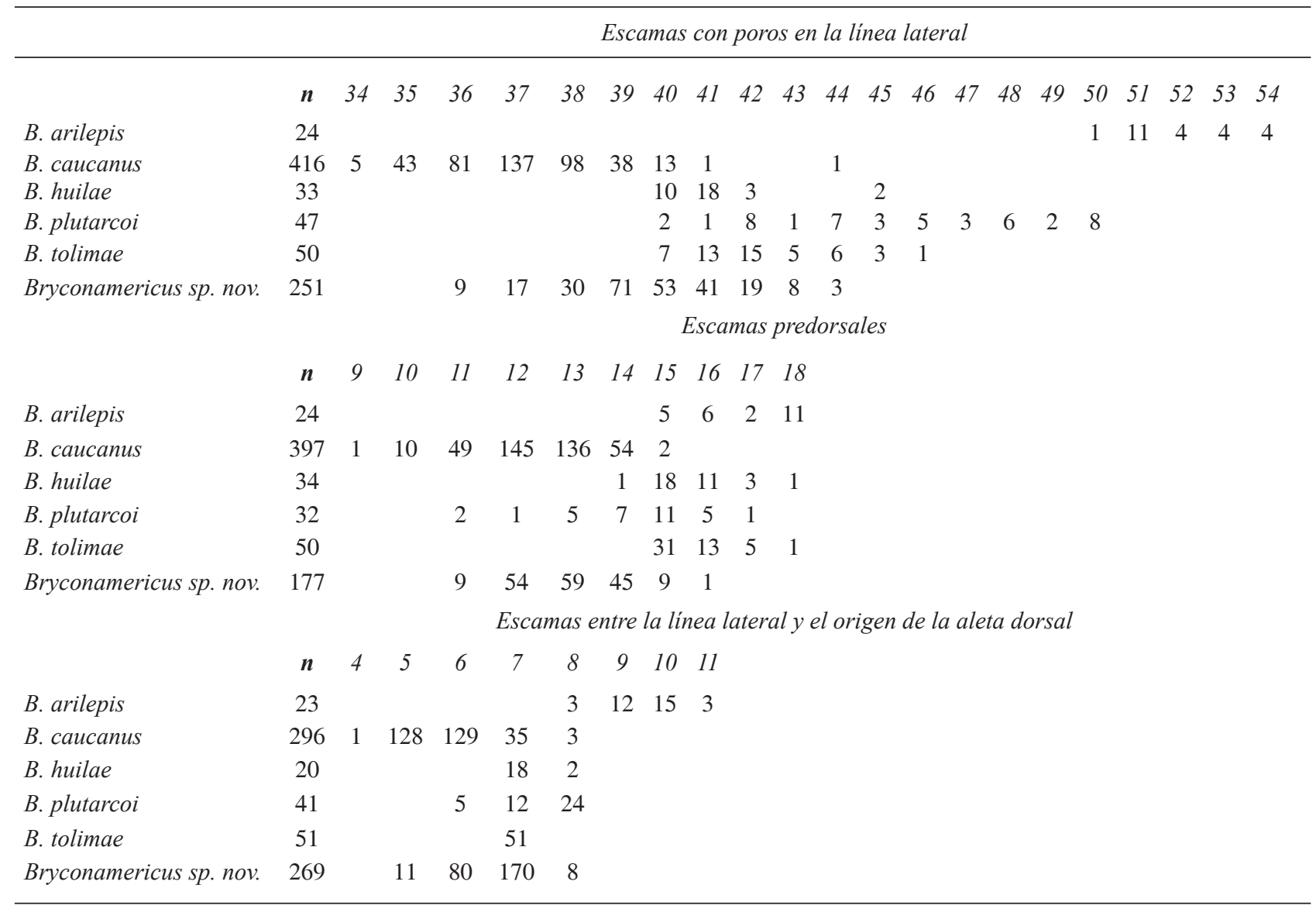


trabajo. Además, B. caucanus, $H$. dentatus y H. decurrens se ubicaron en Bryconamericus (Eigenmann, 1913); según el ICZN (1999), por principio de prioridad, H. dentatus y $H$. decurrens son sinónimos modernos de B. caucanus.

\section{Agradecimientos}

Se recibió financiación de la Universidad del QuindíoVicerrectoría de Investigaciones (proyectos 212 y 304). Al Grupo de Investigación y Asesoría en Estadística de la Universidad del Quindío, en especial a H. Hurtado y D. Galvis por sus generosos comentarios, discusión y sugerencias a versiones de este artículo. Carlos A. García (IUQ) y dos revisores anónimos leyeron versiones del artículo y plantearon valiosas correcciones, críticas y sugerencias. A Donald C. Taphorn (MCNG) por su generosa ayuda, envió las fotos de los tipos depositados en FMNH y corrigió el resumen en inglés.

\section{Literatura citada}

Barriga, R. 1991. Lista de vertebrados del Ecuador. Peces de agua dulce. Politécnica 26:7-88.

Burnaby T. P. 1966. Growth-invariant discriminant functions and generalized distances. Biometrics 22:96-110.

Böhlke, J. 1958. Studies on fishes of the family Characidae.No. 14. A report on several extensive recent collections from Ecuador. Proceedings of the Academy of Natural Sciences of Philadelphia 110:1-121.

Cardona, M., C. Román-Valencia, J. L. Jiménez y H. Hurtado. 1998. Composición y diversidad de los peces de la quebrada San Pablo en alto Cauca, Colombia. Boletín Ecotrópica 32:11-24

Dahl, G. 1971 Los peces del norte de Colombia. Inderena, Bogotá D. C. $391 \mathrm{p}$.

Eigenmann, C. H. 1913. Some results from an ichthyological reconnaissance of Colombia, South America, part II. Indiana University Studies 131:1-31.

Eigenmann, C. H. 1922. The fishes of the northwestern South America including Colombia, Panama and Pacific slopes of Ecuador and Peru, together with appendix upon the fishes of the Rio Meta in Colombia. Memoirs of Carnegie Museum 9:1-348.

Eigenmann, C. H. 1927. The American Characidae. Memoirs of the Museum of Comparative Zoology 43:311-358; 417-428.

Eigenmann, C. H. y W. R. Allen. 1942. Fishes of the western South America: I. The intercordilleran and Amazonian lowlands of Peru, II. The high Pampas of Peru, Bolivia and North Chile, with a revision of the Peruvian Gymnotidae, and of the genus Orestias. The University of Kentucky. p.224-225.

Fink, W. L. y S. H. Weitzman. 1974. The so-called cheirodontin fishes of Central America with descriptions of two new species (Pisces: Characidae). Smithsonian Contributions to Zoology 172:1-46.

Fowler, W. H. 1948. Os peixes de água doce do Brasil. Arquivos de Zoologia, São Paulo 6:1-204.

Fowler, W. H. 1951. Os peixes de água doce do Brasil. Arquivos de Zoologia, São Paulo 6:405-628.

Géry, J. 1962. Notes on the ichthyology of Surinam and other Guiana. The distribution pattern of the genus Hemibrycon, with a description of a new species from Surinam and an incursion in to ecotaxonomy. Bulletin Aquatic Biology 3:65-80.

Géry, J. 1966. A review of certain tetragonopterinae (Characoidei), with the description of two new genera. The Aquarium Journal 37:211-236.

Géry, J. 1972. Contribution a l'etude des poissons characoides de l'equater, avec. une revision du genre Pseudochalceus et la description d'un nouveau genre endemique du rio Cauca en Colombie. Acta Humboldtiana ser. geologica, Paleontologica et Biologica 2:1-112.

Géry, J. 1977. Characoids of the world. T.F.H., Neptune City, New Jersey. 672 p.

González-Díaz, A. A., E. Díaz-Pardo, M. Soria-Barreto y R. Rodiles-Hernández. 2005. Análisis morfométrico de los peces del grupo labialis, género Profundulus (Cyprinodontiformes: Profundulidae), en Chiapas, México. Revista Mexicana de Biodiversidad 76:55-61.

ICZN (Internacional Commission on Zoological Nomenclature). 1999. Internacional code of zoological nomenclature, 4rd ed. Internacional Commission on Zoological Nomenclature, London y University California Press, Berkeley, Los Angeles. $306 \mathrm{p}$.

Jiménez, J. L., C. Román-Valencia y M. Cardona. 1998. Distribución y constancia de las comunidades de peces en la quebrada San Pablo, cuenca del Río La Paila, alto Cauca, Colombia. Actualidades Biológicas 20:21-27.

Langeani, F., Z. M. S. De Lucena, J. P. Lima y F. J. TarelhoPereira. 2005. Bryconamericus turiuba, a new species from the upper Río Paraná system (Ostariophysi: Characiformes). Copeia 2005:386-392.

Lima, F. T. C., L. R. Malabarba, P. A. Buckup, J. F. P. Da Silva, R. P.Vari, A. Harold, R. Benine, O. T. Oyakawa, C. S. Pavaneli, N. A. Menezes, C. A. S . Lucena, M. C. S. L. Malabarba, Z. M. S. Lucena, R. E. Reis, F. Langeani, L. Cassati, V. A. Bertaco, C. Moreira y P. H. F. Lucinda. 2003. Genera Incertae Sedis in Characidae. In Check list of the freshwater fishes of South and Central America, R. E. Reis, S. O. Kullander, y Jr. C. J. Ferraris (eds.). Edipucrs, Porto Alegre, Rio Grande do Sul. p. 106-169.

Leviton, A. E., Jr. R. H. Gibbs, E. Heal y C. E. Dawson. 1985. Standards in herpetology and ichthyology: Part I. Standard symbolic codes for institutional resource collections in herpetology and ichthyology. Copeia 1985:802-832.

Maldonado-Ocampo, J. A., A. Ortega-Lara, J. S. Usma, G. Galvis, F. A. Villa-Navarro, L. V Gamboa, S. Prada-Pedreros y C. Ardila. 2005. Peces de los Andes de Colombia. Instituto de Investigación de Recursos Biológicos Alexander von Humboldt, Bogotá, D. C. 346 p.

Miles, C. 1947. Los peces del río Magdalena. Ministerio de 
Economía Nacional, sección de piscicultura, pesca y caza, Bogotá. 214 p.

Miles, C. 1971. Los peces del río Magdalena, reimpresión. U.T., Ibagué, Tolima. 213 p.

Miles, C. 1973. Estudio económico y ecológico de los peces de agua dulce del Valle del Cauca. Cespedesia 2:9-63.

Miquelarena, A. M. y A. E. Aquino. 1995. Situación taxonómica y geográfica de Bryconamericus thomasi (Teleostei, Characidae). Revista Brasileira. Biología 55:559-569.

Mojica, J. I., C. Castellanos, P. Sánchez-Duarte y C. Díaz. 2006. Peces de la cuenca del río Ranchería, La Guajira, Colombia. Biota Colombiana 7:129-142.

Myers, G. S. 1930. Fishes from the upper Río Meta basin, Colombia. Proceeding Biology Society Washington 43:6571.

Ortega, H. y R. R. Vari. 1986. Annotated checklist of the freshwater fishes of Peru. Smithsonian Contributions to Zoology 437:1-22.

Price, J. L. 1955. A survey of the freshwater fishes of the island of Trinidad. Journal of the Agricultural Society of Trinidad \& Tobago 55:390-416.

Reeves, R. G. y E. Bermingham. 2006. Colonization, population expansion, and lineage turnover: Phylogeography of mesoamerican characiform fish. Biological Journal of the Linnean Society 88:235-255.

Rohlf, J. F. y F. L. Bookstein. 1987. A comment on shearing as a method for "size correction". Systematic Zoology 36:356367.

Román-Valencia, C. 1993. Composición y estructura de las comunidades de peces de la cuenca del río La Vieja, alto Cauca, Colombia. Biología y Educación 3:8-19.

Román-Valencia, C. 1995. Lista anotada de los peces de la cuenca del río La Vieja, Alto Cauca, Colombia. Boletín Ecotrópica: Ecosistemas Tropicales 29:11-20.

Román-Valencia, C. 2001. Descripción de una nueva especie de Bryconamericus (Ostariophysi, Characidae) del alto río Suárez, cuenca del Magdalena, Colombia. Bolletino del Museo Regionale di Scienze Naturali, Torino 18:469-476.

Román-Valencia, C. 2002. Revisión sistemática de las especies del género Bryconamericus (Teleostei: Characidae) de Centroamérica. Revista de Biología Tropical 50:173-192.

Román-Valencia, C. 2003a. Sistemática de las especies colombianas de Bryconamericus (Characiformes, Characidae). Dahlia (Revista de la Asociación Colombiana de Ictiólogos) 6:17-58.

Román-Valencia, C. 2003b. Descripción de tres nuevas especies de Bryconamericus (Pisces: Ostariophysi: Characidae) de Colombia. Memorias de la Fundación La Salle de Ciencias Naturales 155:31-49.

Román-Valencia, C. 2004. Redescripción de Bryconamericus tolimae (Pisces: Characidae), especie endémica del río Combeima, cuenca del río Magdalena, Colombia. Dahlia (Revista de la Asociación Colombiana de Ictiólogos) 7:2327.

Román-Valencia, C. 2005. Sinopsis comentada de las especies del género Bryconamericus (Teleostei: Characidae) de Venezuela y norte del Ecuador, con la descripción de una nueva especie para Venezuela. Memorias de la Fundación La
Salle de Ciencias Naturales 163:27-52.

Román-Valencia, C., P. A. Lehmann y E. Rubio. 1999. Distribución y constancia de los peces del río San Miguel y el Zanjón Bagazal en el Alto río Cauca, Colombia. Actualidades Biológicas 21:163-172.

Román-Valencia, C. y A. Muñoz. 2001. Ecología trófica y reproductiva de Bryconamericus caucanus (Pisces: Characidae). Bolletino del Museo Regionale di Scienze Naturali, Torino 18:459-467.

Román-Valencia, C. y R I. Ruiz-C. 2007. Una nueva especie de pez del género Hemibrycon (Characiformes: Characidae) del Alto Río Atrato, noroccidente de Colombia. Caldasia 29:121-131.

Román-Valencia, C. y D. C. Arcila-Mesa. 2008. Hemibrycon rafaelense, n.sp. (Characiformes, Characidae), a new species from the upper Cauca River with key to Colombian species. Animal Biodiversity and Conservation 31: 67-75.

Román-Valencia, C., D. C. Taphorn y R. I. Ruiz-C. 2008a. Two new Bryconamericus: $B$. cinarucoense $n$.sp. and $B$. singularis n. sp. (Characiformes, Characidae) from the Cinaruco River, Orinoco Basin, with key to all Venezuelan Species. Animal Biodiversity and Conservation 31:15-27.

Román-Valencia, C., J. A. Vanegas-Ríos y R. I. Ruiz-C. 2008 b. Una nueva especie del género Bryconamericus (Ostariophysi: Characidae) del río Magdalena, con una clave para las especies de Colombia. Revista de Biología Tropical 56: en prensa.

Román-Valencia, C., R. I. Ruiz-Calderón y A. Giraldo. 2008c. Dieta y reproducción de dos especies sintópicas: Hemibrycon boquiae y Bryconamericus caucanus (Pisces: Characidae) en la quebrada Boquía, Río Quindío, Alto Cauca, Colombia. Revista Museo Argentino de Ciencias Naturales, n.s. 10:5562.

Román-Valencia, C., J. A. Vanegas-Ríos y R. I. Ruiz-C. En prensa. Una nueva especie del género Bryconamericus (Teleostei: Characidae) del río Fonce, sistema río Magdalena, Colombia. Revista Mexicana de Biodiversidad.

Serra, J. P. y F. Langeani. 2001. Descrição osteológica de Bryconamericus exodon Eigenmann, 1907 (Ostariophysi, Characiformes, Characidae). Teses, Universidade Estadual Paulista, Instituto de Biociências, Letras e Ciências Exatas, Campus de São José do Rio Preto, São Paulo. 21 p.

Serra, J. P. y F. Langeani. 2006. Redescrição e osteologia de Bryconamericus exodon Eigenmann, 1907 (Ostariophysi, Characiformes, Characidae). Biota Neotropica 6:1-14.

Silva, J. F. P. 2004. Two new species of Bryconamericus Eigenmann (Characiformes: Characidae) from southern Brazil. Neotropical Ichthyology 2:5-60.

Taylor, W. R. y G. C. Van Dyke. 1985. Revised procedures for staining and clearing small fishes and other vertebrates for bone and cartilage study. Cybium 9:107-119.

Tortonese, E. 1941-1942. Ricerche ed osservazioni sui Caracidi delle sottofamiglie Tetragonopterinae, Glandulocaudinae e Stethaprioninae (Teleostei, Plectospondyli). Bolletino del Museu Zoologie di Anatomie Comparada, Torino 49:76

Vanegas-Ríos, J. A. 2008. Filogenia y biogeografía de las especies de peces pertenecientes al género Bryconamericus (Characiformes, Characidae) para América Central y 
noroccidente de América del Sur. Trabajo de grado (B. Sc.), Universidad del Quindío, Programa de Biología, Armenia. $97 \mathrm{p}$.

Vari R. P. y D. J. Siebert. 1990. A new, unusually sexually dimorphic species of Bryconamericus (Pisces: Ostariophysi:
Characidae) from the Peruvian Amazon. Proceedings of the Biological Society of Washington 103:516-524.

Weitzman, S.H. 1962. The osteology of Brycon meeki, a generalized characid fish, with an osteological definition of the family. Stanford Icthyological Bulletin 8:1-50.

Apéndice 1. Material examinado

\section{COLOMBIA:}

Bryconamericus caucanus: (FMNH 56230 paratipos, IUQ 335, 336, 337, 340, 341, 342, 344, 346, 351, 352, 378, 379 en Román-Valencia, 2003a). FMNH 56255, 1 ejemplar, Soplaviento, Colombia, Col. C. H. Eigenmann (holotipo $H$. decurrens). Paratipos H. dentatus: FMNH 56252, 5 ejemplares, La Paila, Colombia, Col. C. H Eigenmann; FMNH 56254, 5 ejemplares, Piedra de Moler, Colombia. Col. C. H. Eigenmann, feb 2 de 1912. MBUCV 27914, 15 ejemplares, río Hurtado, afluente del río Cesar, cuenca del río Magdalena, sin fecha. IUQ 349, 113 ejemplares; departamento de Antioquia, municipio de Anorí; La Rosa, río Cauca; oct. 17 de 1988. IUQ 350, 77 ejemplares, departamento de la Guajira; río Ranchería; ago 24 de 1981. IUQ 10, 4 ejemplares, departamento de Quindío, quebrada afluente del río Quindío, ago 9 de 1994. IUQ 227, 2 ejemplares, departamento del Valle, cuenca del río La Paila, septiembre 15 de 1996. IUQ 254, 9 ejemplares, departamento de Quindío, río La Vieja en la Balastrera, hacienda Playa Azul, alto Cauca, jun de 1996. IUQ 272, 1 ejemplar, quebrada San Pablo, afluente río La Paila, Valle del Cauca, alto Cauca, sep 14 de 1996, IUQ 613, 4 ejemplares, departamento de Quindío, río La Vieja, vereda Maravelez, la Tebaida, mar 25 de 1991. IUQ 1269, 3 ejemplares, departamento de Quindío, quebrada El Macho, afluente del río Barragán, jul. 8 de 1996. IUQ 1301, 6 ejemplares, departamento de Caldas, río Chamberri, afluente río Cauca, en la India, Salamina (5² $24^{\prime} 55^{\prime}$ ” N y $75^{\circ} 30^{\prime} 10^{\prime \prime}$ O), jul 2 de 2004. IUQ 856, 35 ejemplares, departamento de Antioquia, quebrada Los Dragos, afluente del río arriba vereda Francisca-Sacantin, Sonsón. IUQ 870, 32 ejemplares, departamento de Antioquia, río Anorí, afluente

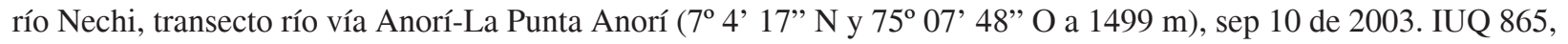
8 ejemplares, departamento de Antioquia, quebrada La Chinca, afluente del río Anorí $200 \mathrm{~m}$ de La Punta, sep 10 de 2003. IUQ 875, 38 ejemplares, departamento de Antioquia, quebrada La Tupia, afluente río Nechi, Anorí, sep. 9 de 2003. IUQ 862, 29 ejemplares, departamento de Antioquia, quebrada La Virgen, afluente río Anorí $100 \mathrm{~m}$ antes de su desembocadura, sector del río vía Anorí, La Punta Anorí, (7 04' 22” N y 75² 07'29” O a 1489 m). IUQ 872, 17 ejemplares, departamento de Risaralda, quebrada El Tigre, hacienda Guadalupe, vereda el Tigre, Pereira, jul 19 de 2005. IUQ 859, 5 ejemplares, departamento de Antioquia, quebrada Serrana, afluente del río Anorí, $200 \mathrm{~m}$ de la vía Anorí, La Punta-Anorí, sep 10 de 2003. IUQ 858, 10 ejemplares, departamento de Antioquia, río Sonsón 100 m antes de la represa, Sonsón, jul 18 de 2004. IUQ 301b, 1 ejemplar (C. y T.), departamento del Quindío, alto río Quindío, alto Cauca, ene 28 de 2000. IUQ 851, 6 ejemplares, río Anoria, La Rosa, bajo Cauca, sistema río Magdalena, oct 17 de 1988. IUQ 861, 2 ejemplares (C. y T.), departamento de Antioquia, cuenca del Magdalena, quebrada La Virgen

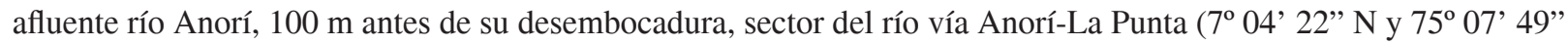
O) 1489 m, sep 10 de 2003. IUQ 863, 2 ejemplares (C. y T.), departamento de Antioquia, cuenca del Magdalena, río Anorí afluente río Nechí, sector río vía Anorí-La Punta (7 4 4 17” N y 7507’ 48” O) 1499 m, sep 10 de 2003; IUQ 864, 2 ejemplares (C. y T.), departamento de Antioquia, cuenca del Magdalena, quebrada La Tupía afluente río Henchí, sep 9 de 2003. IUQ 868, 2 ejemplares (C. y T.), departamento de Antioquia, cuenca del Magdalena, quebrada La Chinca afluente río Anorí, 200 m vía Anorí-La Punta, sep 10 de 2003. IUQ 879, 1 ejemplar (C. y T.), departamento de Antioquia, cuenca del Magdalena, Quebrada Serrana afluente río Anorí, 200 m de la vía Anorí-La Punta, sep 10 de 2003. IUQ 943, 1 ejemplar (C. y T.), departamento de Antioquia, río Sonsón 100 m antes de la represa, Sonsón, cuenca del Magdalena, jul 18 de 2004. IUQ 948, 1 ejemplar (C. y T.), departamento de Antioquia, quebrada Los Dragos, afluente del río Arriba vereda Francisca-Sacantin, Sonsón, cuenca del Magdalena, jul 18 de 2004. IUQ 1202, 1 ejemplar (C. y T.), departamento del Quindío, quebrada Boquia, afluente río Quindío. IUQ 1571, 2 ejemplares (C. y T.) , departamento de Caldas, río Chamberri, afluente río Cauca, en la India, Salamina ( $5^{\circ} 24^{\prime} 55^{\prime \prime} \mathrm{N}$ y $\left.75^{\circ} 30^{\prime} 10^{\prime \prime} \mathrm{O}\right)$. IUQ 1584, 1 ejemplar (C. y T.), departamento de Guajira, río Ranchería, mar Caribe, ago 20 de 1981. Bryconamericus huilae: (IUQ 423 y 424 en Román-Valencia, 2003b). IUQ 947, 2 ejemplares (C. y T.), departamento del Huila, quebrada La Criolla, afluente río Guachino, vereda Guachicos en la vía Pitalito-San Agustín, alto Magdalena, oct 13 de 2001. Bryconamericus tolimae: (Véase IUQ 484, Román-Valencia, 2004). IUQ 1586, 2 ejemplares (C. у T.), 


\section{Apéndice 1. Material}

departamento del Tolima, Municipio de Ibagué, vereda Pastales, 100 m antes de Pastales en la vía Ibagué-Juntas, río Combeima, afluente río Magdalena (4³0'19" N y 75¹7’46” O) 1586 m, dic 27 de 2002 . Bryconamericus plutarcoi: (material tipo IUQ 308, ICNMNH 4886, 4887 en Román-Valencia 2001). IaVH 3123, 12 ejemplares, río Suárez; ene de1994. IUQ 1577, 1 ejemplar (C. y T.), quebrada Santa Rosa de la desembocadura en el río Suárez $100 \mathrm{~m}$ hacia arriba, sistema del río Magdalena (6 26' 09' ' N y 73¹8' 56' O) 825 m, jun 24 de 2001. Bryconamericus arilepis. IUQ 920, 4 ejemplares, quebrada Pontesuela, afluente río Pienta, sistema río Fonce, en la escuela Carrillo, vía a Encino, Charalà, feb 6 de 2004. IUQ 1567, 1 ejemplar (C. y T.), quebrada Pontesuela, afluente río Pienta, sistema río Fonce, en la escuela Carrilo, vía a Encino, Charala, feb 6 de 2004. IUQ 1915, 85 ejemplares, Charalá, vereda La Pontesuela, Magdalena medio, cuenca del río Fonce, quebrada La Pontesuela en la boca de la quebrada Coclina, en la escuela Carrillo, vía Cantera a Encino (6¹0`42” N y los 7309`44”O) 1457 msnm, 17 ene de 2008, C. Román-V. y C. A. García. Bryconamericus sp. nov.: IUQ 919, 63 ejemplares, quebrada La Potrera, afluente río Pienta, cuenca río Fonce, vía Charalà-La laguna, feb 5 de 2004. IUQ 921, 44 ejemplares, quebrada El Barro, afluente río Pienta, 200 m puente vía limites, Encino Charalá, vía el Encino-Charala, feb 6 de 2004. IUQ 923, 41 ejemplares, quebrada La Sanguina, afluente río Pienta, Charalá, feb 5 de 2004. IUQ 926, 22 ejemplares, río Fonce, afluente del río San Gil, vía San Gil, Charalá, oct 31 de 2003. IUQ 927, 35 ejemplares, quebrada Guayawata, afluente del río Mogotes en el puente $100 \mathrm{~m}$ antes de su boca, Mogotes, nov 1 de 2003. IUQ 937, 3 ejemplares (C. y T.), quebrada Guayawata afluente río Mogotes en el puente $100 \mathrm{~m}$ arriba de su boca (6²9' 07' ' N y 72 58' 59', O) 1658 m, en Mogotes, nov 1 de 2003. IUQ 944, 2 ejemplares (C. y T.), río Fonce afluente río San Gil, vía San Gil-Charalá, Charalá, oct 31 de 2003. IUQ 1569, 2 ejemplares (C. y T.), quebrada La Potrera, afluente río Pienta, cuenca río Fonce, vía Charala-La laguna Charalà, feb 5 de 2004 . IUQ 1570, 2 ejemplares (C. y T.), río Mogotes, en la boca quebrada Guayawata $100 \mathrm{~m}$ abajo del puente $1 \mathrm{~km}$ antes de Mogotes, feb 7 de 2004. IUQ 1572, 2 ejemplares (C. y T.), quebrada La Sanguina, afluente río Pienta, Charalá, feb 5 de 2004. IUQ 1573, 2 ejemplares (C. y T.), quebrada El Barro, afluente río Pienta, 200 m puente vía límites, EncinoCharalá, vía el Encino a Charalá. 